\title{
In-line interference mitigation techniques for spectral coexistence of GEO and NGEO satellites
}

\author{
Shree Krishna Sharma*,†, Symeon Chatzinotas and Björn Ottersten \\ Interdisciplinary Centre for Security, Reliability and Trust (SnT), University of Luxembourg L-2721, Luxembourg
}

\begin{abstract}
SUMMARY
The interest towards the deployment of Low Earth Orbit (LEO)/Medium Earth Orbit (MEO) satellite systems in several frequency bands is increasing due to the requirement of low latency for real-time systems and high demand of broadband data. When the number of usable Non-Geostationary (NGEO) satellites, that is, LEO/MEO in space, increases, the frequency coexistence between the NGEO satellite systems with the already existing geostationary (GEO) satellite networks becomes a requisite. In this context, it is crucial to explore interference mitigation techniques between GEO and NGEO systems in order to allow their spectral coexistence. More specifically, in the coexistence scenario of GEO and NGEO satellite networks, in-line interference may be a serious problem, especially in the equatorial region. In this paper, we provide several frequency sharing studies in the context of the coexistence of an NGEO satellite link with another NGEO/GEO satellite link. Furthermore, we carry out interference analysis between GEO and MEO satellite systems considering the case of the O3b satellite system and propose an adaptive power control technique for both the uplink and downlink scenarios in order to mitigate the in-line interference. Moreover, we suggest several cognitive solutions for mitigating the in-line interference and provide future research issues. Copyright (C) 2014 John Wiley \& Sons, Ltd.
\end{abstract}

Received 25 August 2013; Revised 25 February 2014; Accepted 7 August 2014

KEY WORDS: satellite communications; cognitive radio techniques; dual satellite coexistence; O3b satellite system

\section{INTRODUCTION}

Several Satellite Communications (SatComs) systems have been proposed in the literature for the provision of fixed, mobile, interactive and personal services, adopting Geostationary (GEO) and NonGeostationary (NGEO) orbits such as Low-altitude Earth Orbits (LEO) and Medium-altitude Earth Orbits (MEO). Next generation satellite systems require significantly higher spectral efficiency to address the spectrum scarcity problem, and different satellite systems need to coexist within the same spectrum in order to achieve this objective. In this context, cognitive SatComs is a promising candidate providing different opportunities for the spectral coexistence of two satellite networks [1]. GEO satellites utilize a circular orbit above the Earth's equator maintaining the same position relative to the Earth's surface while the positions of NGEO satellites change quite rapidly with time. The main advantages of NGEO satellites in comparison to GEO satellites are reduced free space attenuation, small propagation delay and the reduced cost of in-orbit injection per satellite [2]. Recently, the Other Three Billion (O3b) network has proposed to launch O3b satellites in the MEO of $8062 \mathrm{~km}$ in order to improve the round trip latency as compared to that of the GEO satellite. The O3b network proposes to use parts of the Ka band (uplink: 27.5-30.0 GHz; downlink: 17.8-20.2 GHz) that are also being used by the GEO networks. To facilitate the coordination in the Ka band, International Telecommunication Union-Radiocommunication (ITU-R) footnote 5.523A related to the bands $17.8-19.3 / 28.6-29.1 \mathrm{GHz}$

${ }^{*}$ Correspondence to: Shree Krishna Sharma, Interdisciplinary Centre for Security, Reliability and Trust (SnT), University of Luxembourg L-2721, Luxembourg.

†E-mail: shree.sharma@uni.lu. 
specifies that the coordination is subject to the radio regulations RR 9.11A, that is, priority based on the date of filing. In the rest of the frequency band, Effective Power Flux Density (EPFD) limits specified in the RR Article 22 should be respected for coordination with the already existing satellite systems. Furthermore, the Federal Communications Commission (FCC) has already permitted Teledesic to operate its service links (uplink: 28.6-29.2 GHz; downlink: $18.8-19.3 \mathrm{GHz}$ ) and the gateway terminal links (uplink: 27.6-28.4 GHz; downlink 17.8-18.6 GHz) on a secondary noninterference basis in the Ka band.

In the coexistence scenarios of GEO and NGEO networks, in-line interference may be a serious problem and it arises whenever an NGEO satellite passes through a line of sight path between an earth station and a GEO satellite. This is due to the fact that an earth station that is in line with GEO and NGEO satellites may receive and create interference through its main beam. The in-line interference causes a potential problem to the GEO networks operating near the equator while considering the case of O3b satellites. In this context, exploring efficient techniques to mitigate the in-line interference is a highly relevant and challenging problem for the spectral coexistence of GEO and NGEO satellite networks [3-5].

Cognitive communications is considered a promising candidate for allowing the coexistence of different wireless networks. In the context of SatComs, recent work exploiting spectrum sharing opportunities includes [1, 6-17]. Out of these, the contributions [6, 10, 13, 14, 16, 17] address dual satellite coexistence scenarios. The interference scenario in a satellite system is different from that of the terrestrial systems due to the presence of the on-board antenna that acts as a spatial filter [2]. The cochannel interference mainly arises due to the presence of side-lobes in the on-board antenna radiation pattern, that is, nonideal angular selectivity of the spotbeams and in the radiation patterns of the earth station terminals. In NGEO satellite systems, the relative position of the cochannel spots changes over time due to the constellation dynamics. Due to this, the interference analysis between the systems operating in GEO and NGEO systems becomes more challenging. In [2], several techniques such as spot turnoff, intraorbital plane frequency division and interorbital plane frequency division have been identified in order to avoid or minimize the cochannel interference between GEO and NGEO systems. While considering these techniques, terrestrial Fixed Service (FS) networks operating in the same spectrum should also be taken into account. In the spot turnoff method, one of the two spots is turned off whenever two spots overlap too much. In the intraorbital plane frequency division method, satellites on the same orbital plane are assigned different frequency subsets up to a specified modulo $R$, whereas in the interorbital plane frequency division, the available frequency spectrum is subdivided into as many subsets as the number of orbital planes in such a way that satellites on different orbital planes do not interfere with each other.

\subsection{Types of satellite systems}

Depending on the satellite orbit height (h), the satellite orbits can be classified into LEO, MEO and GEO. The satellites situated at $500<h<2000 \mathrm{~km}$ are LEO satellites, satellites with $5000<h<$ $20000 \mathrm{~km}$ are MEO satellites and the satellites with $h=35800 \mathrm{~km}$ are GEO satellites. Since the satellite footprint decreases in size as the orbit becomes lower, LEO and MEO systems require larger constellations than the GEO satellites in order to achieve the global coverage and avoid data transmission delays. However, low transmit power is sufficient for LEO and MEO satellite systems because of the shorter average distance between the earth station terminal and the satellite [2]. The LEOs can be further subdivided into big LEO and little LEO categories. The big LEOs can offer voice, fax, telex, paging and data services, whereas the little LEOs can provide only data services, either real-time services or store and forward services [18]. The MEO constellations have some advantages and compromises both the LEO and GEO constellations. They require limited number of satellites to achieve wide coverage with intermediate values of the elevation angles. The values of free space attenuation and propagation delay are also intermediate to those of LEO and GEO values.

In the context of MEO satellites, the O3b network has proposed the O3b constellation of 12 to 20 satellites in a circular MEO at a distance of about $8062 \mathrm{~km}$ from the Earth. Out of which four satellites have been already launched in the operational orbits [19]. The round trip delay of the O3b satellite is $120 \mathrm{~ms}$ as compared to $500 \mathrm{~ms}$ of the GEO satellite. This becomes highly advantageous for enhancing the quality of telephone calls and data throughput using satellite networks. The main advantages of O3b 
network are low latency, high capacity of $1.2 \mathrm{Gbps}$, competitive pricing, easily and quickly deployable structure [20]. The beam footprints have a diameter of about $600 \mathrm{~km}$ on the Earth surface between $45^{\circ}$ North and $45^{\circ}$ South and can be dynamically steered as the satellite moves in order to cover the required areas and skip over the unpopulated areas. At the initial stage, the $\mathrm{O} 3 \mathrm{~b}$ network has planned to place eight satellites at $45^{\circ}$ apart, orbiting around the equator in a noninclined orbit. As noted in [20], the maximum permissible power for satellite earth station around $18 \mathrm{GHz}$ with $0.01 \%$ unavailability is $-146 \mathrm{dBW} / \mathrm{MHz}$. The detailed parameters for O3b network have been provided in Table III.

\subsection{Interference mitigation techniques for coexistence of GEO and NGEO systems}

In this subsection, we provide a review of interference mitigation techniques for the coexistence of an NGEO satellite system with the GEO satellite systems operating in the same spectrum. Furthermore, we provide different ITU-R regulations related to the interference analysis between GEO and NGEO satellite systems in Table I.

The contribution in [21] specifies the following techniques that are commonly used to facilitate spectrum sharing between GEO and NGEO satellites.

(1) Spatial isolation (e.g., GEO orbital slot separation)

(2) Geographical separation between satellite earth terminals

(3) Time/frequency/code isolation

(4) Frequency band segmentation or band planning

(5) Minimum look angle restrictions for sharing between earth terminals and the terrestrial FS links

(6) GEO arc avoidance for NGEO sharing with GEO Fixed Satellite Services (FSS) and GEO Broadcasting Satellite Services (BSS)

(7) Co-coverage avoidance schemes (e.g., National Oceanic and Atmospheric Administration (NOAA) and Little LEOs)

Table I. Related ITU-R recommendations.

\begin{tabular}{|c|c|}
\hline ITU-R recommendations & Description \\
\hline ITU-R S.1419 & $\begin{array}{l}\text { Suggests interference mitigation techniques to facilitate } \\
\text { coordination between NGEO and GEO FSS networks in the bands } \\
19.3-19.7 \mathrm{GHz} \text { and } 29.1-29.5 \mathrm{GHz}\end{array}$ \\
\hline ITU-R S.1323-2 & $\begin{array}{l}\text { Provides the maximum permissible levels of interference in a } \\
\text { satellite network (GEO/FSS, NGEO/FSS, NGEO/MSS feeder links) }\end{array}$ \\
\hline ITU-R S.1428 & $\begin{array}{l}\text { Provides reference earth station radiation patterns for use in } \\
\text { interference assessment involving NGEO satellite networks in } \\
\text { frequency bands between } 10.7 \text { and } 30 \mathrm{GHz}\end{array}$ \\
\hline ITU-R S.1255 & $\begin{array}{l}\text { Provides the recommendation on the use of adaptive uplink power } \\
\text { control to mitigate co-directional interference between GEO FSS } \\
\text { networks and feeder links of NGEO MSS networks as well as } \\
\text { between GEO FSS networks and NGEO FSS networks }\end{array}$ \\
\hline ITU-R S.672-4 & $\begin{array}{l}\text { Provides the satellite antenna radiation patterns for the GEO FSS } \\
\text { satellites }\end{array}$ \\
\hline ITU-R S.1528 & $\begin{array}{l}\text { Provides the satellite antenna radiation patterns for NGEO FSS } \\
\text { satellites operating below } 30 \mathrm{GHz}\end{array}$ \\
\hline ITU RR No 5.523A & $\begin{array}{l}\text { Provides a specific regulatory framework for NGEO FSS systems } \\
\text { with regard to GEO systems in the } 18.8-19.3 \text { and } 28.6-29.1 \\
\text { GHz bands }\end{array}$ \\
\hline ITU-R RR No. 22 & $\begin{array}{l}\text { Contains EPFD limits for NGEO FSS systems in order to protect } \\
\text { GEO FSS networks from unacceptable interference }\end{array}$ \\
\hline ITU-R S.1431 & $\begin{array}{l}\text { Provides different mitigation techniques in order to avoid the in-line } \\
\text { interference between NGEO satellites }\end{array}$ \\
\hline ITU-R S. 1325 & $\begin{array}{l}\text { Provides the methodologies for determining statistics of short term } \\
\text { interference between co-frequency, co-directional NGEO FSS } \\
\text { systems and other NGEO FSS systems or GEO FSS networks }\end{array}$ \\
\hline
\end{tabular}


The recommendation ITU-R S.1431 provides several interference mitigation techniques in order to avoid the in-line interference between NGEO satellites, and these techniques are also applicable for the coexistence of GEO and NGEO satellites. Furthermore, the recommendation ITU-R S.1325 provides the methodologies for determining statistics of short-term interference between co-frequency, co-directional NGEO FSS systems and other NGEO FSS systems or GEO FSS networks. On the basis of these recommendations, the following mitigation strategies can be used in order to mitigate the in-line interference.

(1) GEO arc avoidance based on the latitude. The NGEO satellite systems can use Exclusion Zone (EZ)-based techniques that prevent the coupling between the main beam of their satellites and the main beam of the GEO earth station. An EZ can be defined in terms of angular separation with respect to the equatorial plane.

(2) GEO arc avoidance based on discrimination angle between NGEO satellite and GEO arc. The NGEO systems can implement the GEO arc avoidance strategy by switching off the beams when any earth point within a cell observes an angular separation between the GEO arc and an NGEO satellite of less than a certain predefined angle.

(3) Satellite diversity. This implies performing a handover process due to selection of another satellite for interference avoidance. The use of satellite diversity can be considered as a mitigation technique to avoid main beam to main beam interference by switching traffic to an alternative satellite in view whenever such in-line events occur.

(4) Avoidance without switching to another satellite. Another option for NGEO FSS operators is to cease transmission without switching to another satellite and accept the outage/loss of coverage when a near in-line event occurs.

(5) Satellite selection strategies. The algorithm chosen for satellite selection by a given NGEO FSS system may enhance the ability of that system to share with other NGEO and GEO systems. In general, earth stations communicate with the satellite observed at the highest elevation angle. If a system choose to select a satellite that has the largest angular discrimination with respect to other NGEO FSS satellites, the sharing situation can be improved at the expense of added complexity and/or reduced capacity in system operation.

(6) Satellite antenna side-lobes. The use of low side-lobe antennas in the NGEO FSS satellite may reduce the amount of interference to and from the main beam of GEO earth station antennas in the case of in-line interference when the NGEO satellite is serving a different area than the location of the earth station.

(7) Earth station antenna side-lobes. The use of low side-lobe antennas on NGEO earth terminals decreases the interference to GEO satellite systems on the earth to space link and allows for a smaller avoidance angle.

(8) Frequency channelization. The process of subdividing the licensed bands into smaller bands can be defined as frequency channelization. In this scheme, each sub-band can be assigned to a separate beam that is spatially separated from its nearest co-frequency beam in order to enhance Carrier to Interference (C/I) levels.

\subsection{Scenario and contributions}

The coexistence of NGEO and GEO FSS satellite systems can enhance the overall spectral efficiency of satellite systems by making efficient use of the allocated spectrum in both temporal and spatial domains. In this context, different coexistence techniques can be explored in the normal forward/return mode and reverse mode scenarios [1]. Depending on the coexistence in forward or reverse modes, the following scenarios can be considered.

- LEO/MEO and GEO coexistence in the Ka band with forward band sharing (GEO forward link, LEO/MEO forward link)

- LEO/MEO and GEO coexistence in the Ka band with reverse band sharing (GEO forward link, LEO/MEO return link)

- LEO/MEO and GEO coexistence in the Ka band with forward band sharing (GEO return link, LEO/MEO return link)

- LEO/MEO and GEO coexistence in the Ka band with reverse band sharing (GEO return link, 


\section{LEO/MEO forward link)}

It should be noted that as the number of usable NGEO satellite systems in space increases, the need for frequency coexistence between the NGEO satellite systems with the already existing satellite networks increases rapidly. This coexistence can be in space and time domains or any other possible domains such as polarization, radiation pattern and others. The interference environment generated by NGEO satellite systems is not completely known yet, and the studies have been conducted with the purpose of examining the feasibility of frequency sharing between other services and NGEO satellite systems [3, 22-24].

As mentioned in Section 1.2, the recommendation ITU-R S.1325 provides different strategies such as GEO arc avoidance based on the latitude and based on the discrimination angle between NGEO satellite and the GEO arc. For the GEO arc avoidance based on the latitude, an EZ of $\theta^{\circ}$ can be defined with respect to the equatorial plane, and for another method based on the discrimination angle, the minimum discrimination angle $\alpha^{\circ}$ is required. With the knowledge of these values, different mitigation strategies, such as satellite switching, spot turn off, and others, can be applied. However, it remains an open challenge to find out the optimum values of $\theta^{\circ}$ and $\alpha^{\circ}$. In this context, it is highly possible to operate NGEO earth stations within the GEO EZ by applying a power control technique in such a way that the aggregate interference towards the GEO satellite is below the interference threshold of the GEO satellite.

Although the coexistence of GEO and NGEO satellites have been discussed in the literature by analyzing the interference mechanism between these systems by using different simulation softwares such as Visualyse [25], our approach in this paper is to propose cognitive techniques that will allow these two systems to coexist with better interference management. We consider the scenarios of both GEO and NGEO networks operating in either the normal return mode or the normal forward mode with the GEO satellite as the primary and the NGEO satellite as the secondary. Furthermore, we consider the coexistence of a GEO satellite link operating in the Ka band and an $\mathrm{O} 3 \mathrm{~b}$ satellite link as a use case of the MEO satellite link. In this paper, we focus on the equatorial region for carrying out interference analysis since the $\mathrm{O} 3 \mathrm{~b}$ network causes harmful interference to the GEO network in this region. The main problem that arises in the coexistence of GEO and NGEO networks is the in-line interference event as mentioned earlier. Although this event can be predetermined and avoided by using proper planning considering the constellation geometries, the performance of the primary system may be affected due to limited dynamicity of these methods. Furthermore, the Quality of Service (QoS) of the NGEO system may not be guaranteed while trying to mitigate in-line interference with these static methods.

In the above context, we propose an adaptive power control technique at the NGEO terminal for uplink transmissions and at the NGEO satellite for downlink transmissions. In the proposed technique, the required transmission power is determined to control interference towards the victim receiver, that is, GEO satellite in the uplink transmission and the GEO earth station terminal in the downlink transmission, taking into account of the interference threshold of these victim receivers as well as the required QoS for the NGEO link. Furthermore, we propose different coordinated and uncoordinated cognitive techniques that can be explored further for their practical feasibility. We summarize the contributions of this paper in the following points:

- The link budget parameters of both the networks are presented, and interference analysis between GEO and MEO satellite networks has been carried out.

- The detailed description of frequency sharing studies carried out in the context of GEO/NGEO coexistence is provided.

- An underlay mode of cognitive technique based on power control is proposed in order to combat the in-line interference towards the primary receiver (i.e., GEO satellite in the uplink scenario and the GEO earth station in the downlink).

- Several cognitive techniques are discussed in order to allow the coexistence of GEO and NGEO satellites in an effective way and future research challenges in this domain are identified.

The remainder of this paper is organized as follows: Section 2 reviews frequency sharing studies carried out in the context of the coexistence of GEO and NGEO systems. Section 3 presents the 
spectral coexistence of GEO and MEO satellite systems considering the O3b system as a use case and proposes an adaptive power control technique for the uplink and downlink coexistence scenarios. Section 4 evaluates the system performance with the help of numerical results and further provides a methodology to calculate the NGEO capacity. Section 5 includes further discussion on the considered coexistence and provides future research issues. Furthermore, this section discusses several cognitive solutions including coordinated, dynamic and combined approaches. Section 6 concludes the paper.

\section{FREQUENCY SHARING STUDIES BETWEEN GEO AND NGEO SYSTEMS}

The GEO satellites utilize a circular orbit above the Earth's equator and they maintain the same position relative to the Earth's surface, whereas the NGEO satellites generally have orbits with varying altitudes and positions. It is generally understood that GEO and NGEO satellite networks operating on the same spectrum are not technically compatible without employing some kind of interference mitigation mechanisms by one or the other satellite. Therefore, different frequency bands are generally allocated to each type of satellite service on a primary basis. In this context, the ITU's 1995 World Radiocommunication Conference (WRC-95) has allocated the frequency bands $18.8-19.3 \mathrm{GHz}$ (space to Earth) and 28.6-29.1 GHz (Earth to space) to the GEO and NGEO FSS networks on the co-primary basis [26].

In GEO satellite systems, the propagation distances from the earth to the satellite and from the satellite to the earth are relatively fixed with respect to the Earth's surface. In this case, the interference paths between one GEO network and another GEO network are fixed. Generally, GEO FSS networks are designed in such a way that frequencies can be reused by satellites spaced $3^{\circ}$ apart. According to ITU-R's definition, circular orbits of geostationary height and having plane inclinations up to $5^{\circ}$ can be regarded as quasi-geostationary [22]. In such orbits, the signal and the interference paths between them may vary to a small extent, but not sufficiently to have a major effect on the system performance while using frequency sharing. However, the positions of LEO or MEO satellites change quite rapidly with time with respect to the Earth's surface. For example, the angular velocity of a satellite in a circular orbit of height $1000 \mathrm{~km}$ is about $3.42 \% \mathrm{~min}$, compared with the $0.25 \%$ min angular velocity of the earth, that is, of any GEO satellite [22].

While reusing spectrum between GEO and NGEO networks, forward band sharing and reverse band sharing can be considered [23]. In the forward band sharing, the same uplink and downlink frequencies are shared by both the NGEO link and the GEO link, whereas in the reverse band sharing, the same uplink and downlink frequencies are shared in reverse way. In the forward band sharing, the following interference paths exist.

- Interference from the NGEO gateway station to the GEO satellite

- Interference from the NGEO satellite to the GEO earth station

- Interference from the GEO earth station to the NGEO satellite

- Interference from the GEO satellite to the NGEO gateway station

- Interference from one NGEO gateway station to another NGEO satellite

- Interference from one NGEO satellite to another NGEO gateway station

Similarly, in reverse band scenario, the following interference paths exist [23]:

- Interference from the NGEO satellite to the GEO satellite

- Interference from the NGEO gateway station to the GEO earth station

- Interference from the GEO satellite to the NGEO satellite

- Interference from the GEO earth station to the NGEO gateway station

- Interference from one NGEO gateway station to another NGEO gateway station

- Interference from one NGEO satellite to another NGEO satellite

The excessive interference occurs whenever an NGEO satellite passes through a line of sight path between an earth station and the GEO satellite [22]. An earth station may fall in line with a GEO satellite and an NGEO satellite, and the earth station will both receive and create interference through its main beam. This interference can be treated as in-line interference. If it is a GEO network station, it will receive interference from and interfere with the NGEO satellite. If it is an NGEO network 
feeder station, it will receive interference from and interfere with the GEO satellite. For other earth stations in the GEO and NGEO systems, the interference enters and leaves the earth stations through the side-lobes of their antenna patterns. In this case, the levels of interference to and from the earth stations depend on the side-lobe levels of the antenna patterns. Although the abovementioned in-line interference condition may occur in the forward band sharing, this condition does not occur for reverse band sharing. In [22], the following methods have been specified to protect GEO networks from in-line interference in the context of coexistence of feeder links of NGEO and GEO networks.

- Designing the NGEO feeder links in such a way that the percentage of outage is within the specified limit.

- Turning off the transmitters whenever the antenna axis of NGEO satellites moves within a given angular distance of the GEO.

- Allocating an exclusive band within the FSS allocation bands for NGEO feeder links.

- Using reverse-band in NGEO feeder links than that of GEO feeder links.

Most of the existing methods to assess interference involving NGEO satellite networks are based on direct computer simulation using Visualyse software of Transfinite systems [25]. These methods are usually time consuming and require a lengthy simulation run each time when some of the system and network parameters are changed. Furthermore, in practical situations involving a large number of earth stations and the NGEO satellites, these methods may require a very long simulation run time to produce statistically significant results. In this context, in [4], an analytical approach to assess interference involving NGEO satellite networks has been considered. The concept used in [4] is that the probability of having the satellites of a NGEO system in a given set of positions (defined by the position of a reference satellite and the type of motion of the reference satellite: ascending or descending mode) is calculated analytically and not estimated as in simulation procedures.

The simulation results in [24] indicate a positive potential for sharing between the GEO FSS and the NGEO satellite high rate links with sufficient site separation between the respective earth Stations. In [27], the following two different scenarios have been considered for analyzing the interference between GEO and NGEO satellite networks: (i) downlink interference from the NGEO constellation into the FS system and (ii) downlink interference from the FS system to the NGEO earth station considering a random FS link. Consequently, the protection area in order to protect earth station from FS link interference has been defined. The contribution in [3] studies the effect of interference from the NGEO link into the GEO link using Bit Error Rate (BER) performance analysis. Consequently, the effect of the separation angle between GEO and NGEO and the combined effect of the number of interfering satellites and separation angle have been considered in order to analyze the effect of interference generated by an NGEO link to the GEO link. Furthermore, the contribution in [28] presents the theoretical expressions for calculating the $\mathrm{C} / \mathrm{I}$ ratio both for the uplink and the downlink in NGEO satellite systems.

Integrating different satellite constellations is an important aspect of moving towards the next generation satellite networks. However, most of the existing satellite systems have been operating independent of one another. This provides an opportunity to improve the satellites system performance and create the potential for their new applications. In this context, a multilayered satellite network consisting of multiple satellite constellations having different orbital altitudes has been studied in $[29,30]$ as a space core network, which delivers traffic to any point on the earth. The main advantage of the multilayered satellite networks is that they have the combined benefits of all the networks, that is, the wider coverage area served by upper layers and the shorter propagation delay provided by the lower layers. Furthermore, from the point of fair and efficient utilization of network resources, wide coverage areas of satellites having higher altitudes are preferred for averaging the load of each satellite. In [31], a typical example of two-layered satellite networks has been considered and the load balancing issue with the provision of QoS by using the advantage of the interconnection between layers has been discussed.

\section{SPECTRAL COEXISTENCE OF MEO AND GEO SATELLITES}

Currently, according to ITU RR No 5.523A, NGEO satellite systems can use the bands 17.8-18.6 $\mathrm{GHz}, 19.7-20.2 \mathrm{GHz}, 27.5-28.6 \mathrm{GHz}$ and $29.5-30 \mathrm{GHz}$ in the primary basis by respecting the EPFD 
limits in order to protect the GEO systems. It can be noted that the adjacent bands $18.8-19.3 \mathrm{GHz}$ and 28.6-29.1 GHz bands have been allocated to GEO satellites in the primary basis. By using suitable cognitive techniques, these bands can be shared by GEO and NGEO systems for enhancing the overall spectral efficiency of satellite systems.

As mentioned in Section 1, the O3b network proposes to use parts of the Ka band that are also used by GEO networks. It can be observed that the frequencies used in the O3b are in the following bands [32]: uplink: $27.5-30.0 \mathrm{GHz}$ and downlink: $17.8-19.3 \mathrm{GHz}(19.3-20.2 \mathrm{GHz}$ is planned for later use). Since the frequencies in this range have been already allocated to GEO networks, a number of interference paths exists while sharing these bands by the O3b network. For proper sharing of these bands, it should be guaranteed that the EPFD limits within the specified band do not exceed the prescribed limit by ITU-R. Figures 1 and 2 present the in-line interference condition in the forward normal mode and the return normal mode, respectively. The following interference paths can be considered for the coexistence of O3b network and GEO networks [32].

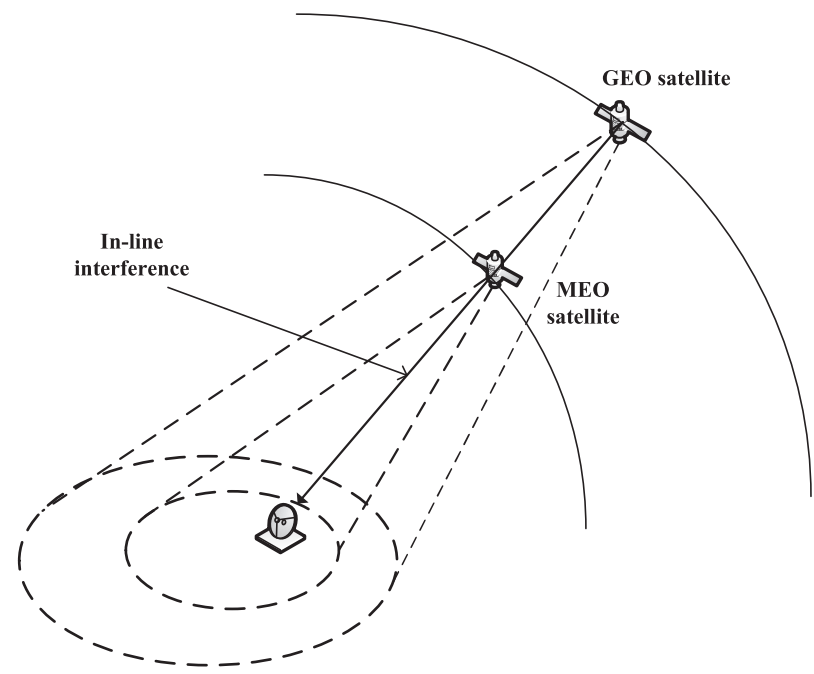

Figure 1. In-line interference in forward normal mode (The SAT terminal can be MEO/GEO terminal and interference paths can be from GEO/MEO satellites, respectively).

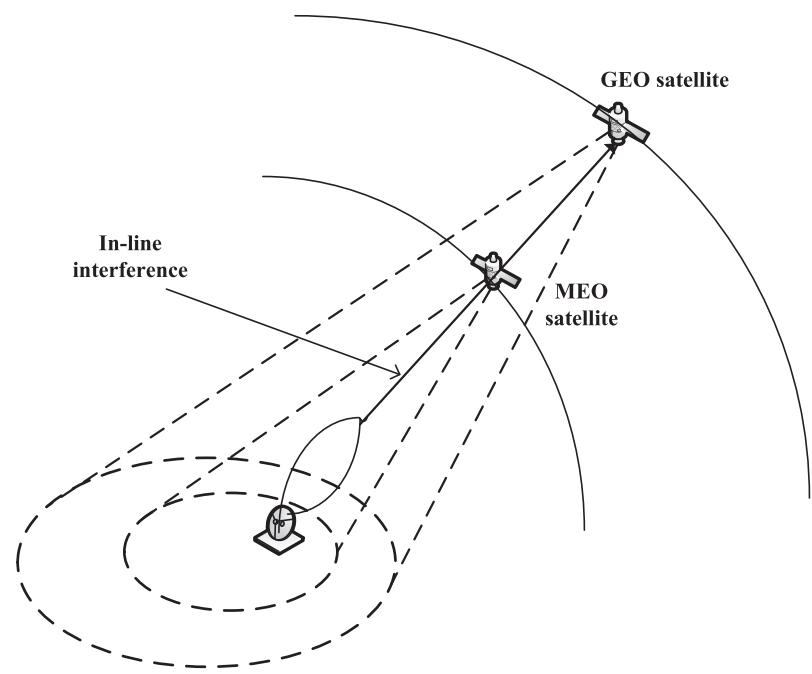

Figure 2. In-line interference in return normal mode (The SAT terminal can be MEO/GEO terminal and interference path can be towards GEO/MEO satellites, respectively). 
- From O3b uplink to the GEO uplink

- From O3b downlink to the GEO downlink

- From O3b downlink to the GEO uplink

- From GEO uplink to the O3b uplink

- From GEO downlink to the O3b downlink

- From GEO uplink to the O3b downlink

It can be noted that for GEO satellite networks serving earth stations at high latitudes, the occurrence of in-line event never occurs, therefore not resulting in any harmful interference to GEO networks. However, for GEO satellite networks serving earth stations near the equator, it can be observed that there could be a potential problem as the O3b satellite may fall in-line between the GEO satellite and an earth station on the equator [32]. The radiation patterns of the earth station antennas play an important role in interference analysis and mitigation between two satellite systems. We assume that the earth stations are equipped with parabolic reflector type antennas with a radiating aperture. For an earth station antenna, the important parameters for characterizing the radiation of the main lobe are the gain, the angular beamwidth and the polarization isolation.

\subsection{Downlink coexistence analysis}

In this scenario, we consider both the GEO and NGEO satellite links operating in the normal forward mode as shown in Figure 3. There exist the following two interference links: (i) from the NGEO satellite to the GEO earth station and (ii) from the GEO satellite to the NGEO earth station. We consider that the GEO satellite is already in operation and the NGEO satellite link is to be deployed in the same spectrum. In this case, the link budget of the NGEO link can be adjusted by taking into account of the interference caused by the GEO satellite to the NGEO link. Therefore, we only consider the interfering link from the NGEO satellite to the GEO earth station. To make the analysis simpler, we consider a single NGEO satellite operating in the same frequency as that of the GEO satellite. ${ }^{\ddagger}$

3.1.1. Problem statement. In this work, we target to solve the following issues for the considered downlink coexistence scenario.

- The downlink transmission from the NGEO satellite may cause interference to the receiver of the GEO earth station. The value of interference to noise ratio, that is, I/N at the GEO earth station, should not exceed the tolerance level of $I / N$.

- The sum rate of the NGEO satellite link should be sufficient to achieve the desired QoS. Increasing the transmit power at the NGEO satellite may enhance the quality of the NGEO link but it may cause interference to the GEO link operating in the same frequency.

- Furthermore, the power on the onboard unit of the NGEO satellite is limited. Therefore, it is necessary to minimize the transmitted power while satisfying the above two conditions.

3.1.2. Proposed power control in the downlink. Let $P_{t n s}$ be the transmit power of the NGEO satellite and $W$ be the transmission bandwidth. Let $\theta_{1}$ be the off boresight angle of the transmitter (NGEO satellite) in the direction of the receiver and $\theta_{2}$ be the off boresight angle of the receiver (GEO earth station) in the direction of the transmitter. We consider $G_{t n s}$ be the gain of the transmit antenna at the NGEO satellite and the $G_{r n e}$ be the gain of the receive antenna at the NGEO earth station. It should be noted that the gain is a function of the off boresight angle and its maximum at the boresight angle; that is, $G_{t n s}(0)$ represents the maximum gain of the transmit antenna of the NGEO satellite and $G_{r n e}(0)$ denotes the maximum gain of the receive antenna of the NGEO earth station. Furthermore, we consider $d_{n n}$ to be the distance between the NGEO station and the NGEO satellite and $d_{n g}$ be the distance

\footnotetext{
${ }^{\ddagger}$ However, the case of the presence of multiple NGEO stations can be straightforwardly incorporated in the analysis. 


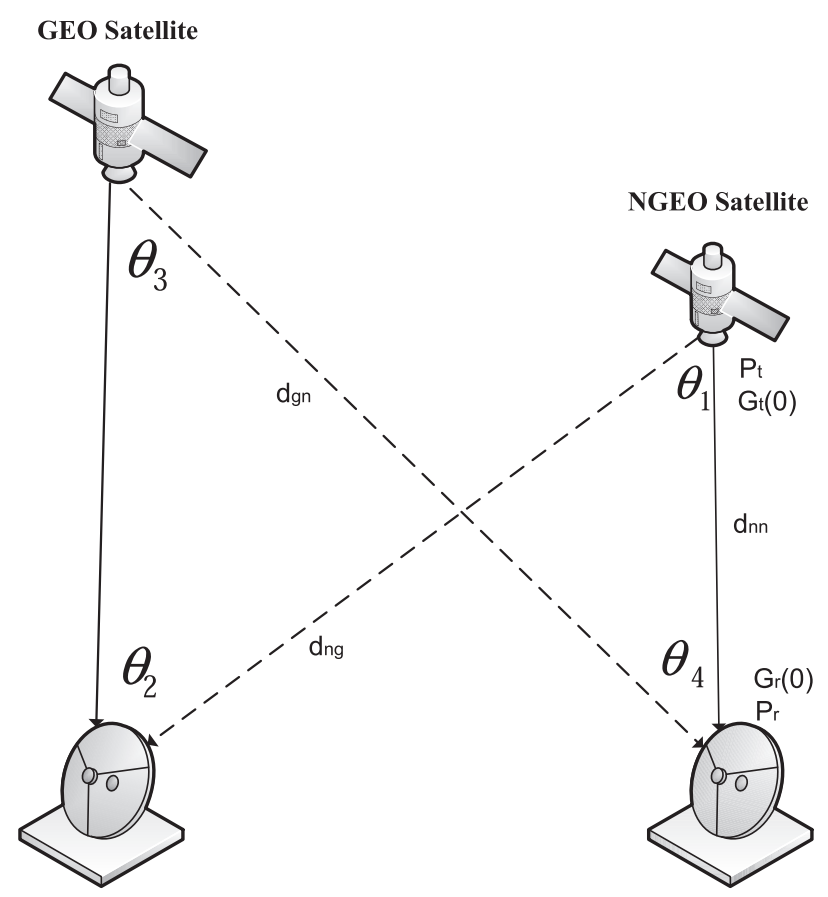

Figure 3. Desired and interference links in the downlink coexistence of GEO and NGEO satellite networks.

between the NGEO satellite and the GEO earth station. The received power at the NGEO earth station can be written as

$$
P_{r n e}=P_{t n s}\left(d_{n n}\right) G_{t n s}(0) G_{r n e}(0)\left(\frac{\lambda}{4 \pi d_{n n}}\right)^{2},
$$

where $P_{t n s}\left(d_{n n}\right)$ is the transmit power required to close the link when the distance between the NGEO station and the NGEO satellite is $d_{n n}$. The expression for Carrier to Noise ratio $(C / N)$ at the NGEO earth station can be expressed as

$$
C / N=\frac{P_{r n e}}{K T_{r n e} W}=\frac{P_{t n s}\left(d_{n n}\right) G_{t n s}(0) G_{r n e}(0)}{K T_{r n e} W}\left(\frac{\lambda}{4 \pi d_{n n}}\right)^{2},
$$

where $K=1.38 \times 10^{-23} \mathrm{~W} /(\mathrm{Hz} \mathrm{K})$ is Boltzmann's constant and $T_{r n e}$ is the receive noise temperature of the NGEO earth station antenna. Furthermore, the interference to noise ratio $(I / N)$ at the GEO earth station due to the presence of NGEO link can be written as

$$
I / N=\frac{P_{t n s}\left(d_{n n}\right) G_{t n s}\left(\theta_{1}\right) G_{r g e}\left(\theta_{2}\right)}{K T_{r g e} W}\left(\frac{\lambda}{4 \pi d_{n g}}\right)^{2},
$$

where $G_{t n s}\left(\theta_{1}\right)$ and $G_{\text {rge }}\left(\theta_{2}\right)$ are the gains of transmit antenna at the NGEO satellite towards the $\theta_{1}$ direction (from the boresight direction) and of the receive antenna at the GEO earth station towards the $\theta_{2}$ direction (from the boresight direction), respectively, and $T_{r g e}$ is the receive noise temperature of the GEO earth station antenna. In order to address the considered problems, the following optimization problem can be formulated:

$$
\begin{array}{r}
\min P_{t n s}\left(d_{n n}\right) \\
\text { subject toC } / N \geqslant C_{0} / N_{0}, \\
I_{g e o} \leqslant I_{t h},
\end{array}
$$


where $I_{t h}$ is the tolerable interference threshold of the GEO satellite and $I_{g e o}$ is the interference received by the GEO earth station due to NGEO downlink transmission. The above optimization problem can also be written in the following form.

$$
\begin{gathered}
\min P_{t n s}\left(d_{n n}\right) \\
\text { subject to } \frac{P_{t n s}\left(d_{n n}\right) G_{t n s}(0) G_{r n e}(0)}{K T_{r n e} W}\left(\frac{\lambda}{4 \pi d_{n n}}\right)^{2} \geqslant C_{0} / N_{0 r n e} \\
\frac{P_{t n s}\left(d_{n n}\right) G_{t n s}\left(\theta_{1}\right) G_{r g e}\left(\theta_{2}\right)}{K T_{r g e} W}\left(\frac{\lambda}{4 \pi d_{n g}}\right)^{2} \leqslant I_{t h} / N_{0 r g e} .
\end{gathered}
$$

Considering the noise temperature does not change over the time at transmit and receive antennas; that is, noise powers $K T_{r g e} W=N_{0 r g e}$ and $K T_{r n e} W=N_{0 r n e}$ remain same. The above problem can be modified into the following:

$$
\begin{gathered}
\min P_{t n s}\left(d_{n n}\right) \\
\text { subject to } P_{t n s}\left(d_{n n}\right) G_{t n s}(0) G_{r n e}(0)\left(\frac{\lambda}{4 \pi d_{n n}}\right)^{2} \geqslant C_{0} \\
P_{t n s}\left(d_{n n}\right) G_{t n s}\left(\theta_{1}\right) G_{r g e}\left(\theta_{2}\right)\left(\frac{\lambda}{4 \pi d_{n g}}\right)^{2} \leqslant I_{t h} .
\end{gathered}
$$

The SINR at the NGEO earth station considering the interference from the GEO satellite can be written as

$$
S I N R_{d}=\frac{P_{t n s} G_{t n s}(0) G_{r n e}(0)\left(\frac{\lambda}{4 \pi d_{n n}}\right)^{2}}{P_{t g s} G_{t g s}\left(\theta_{3}\right) G_{r n e}\left(\theta_{4}\right)\left(\frac{\lambda}{4 \pi d_{g n}}\right)^{2}+K T_{r n e} W},
$$

where $\theta_{3}$ is the offset angle of the GEO transmitting satellite antenna in the direction of the NGEO earth station and $\theta_{4}$ is the offset angle of the NGEO earth station receiving antenna in the direction of the GEO satellite.

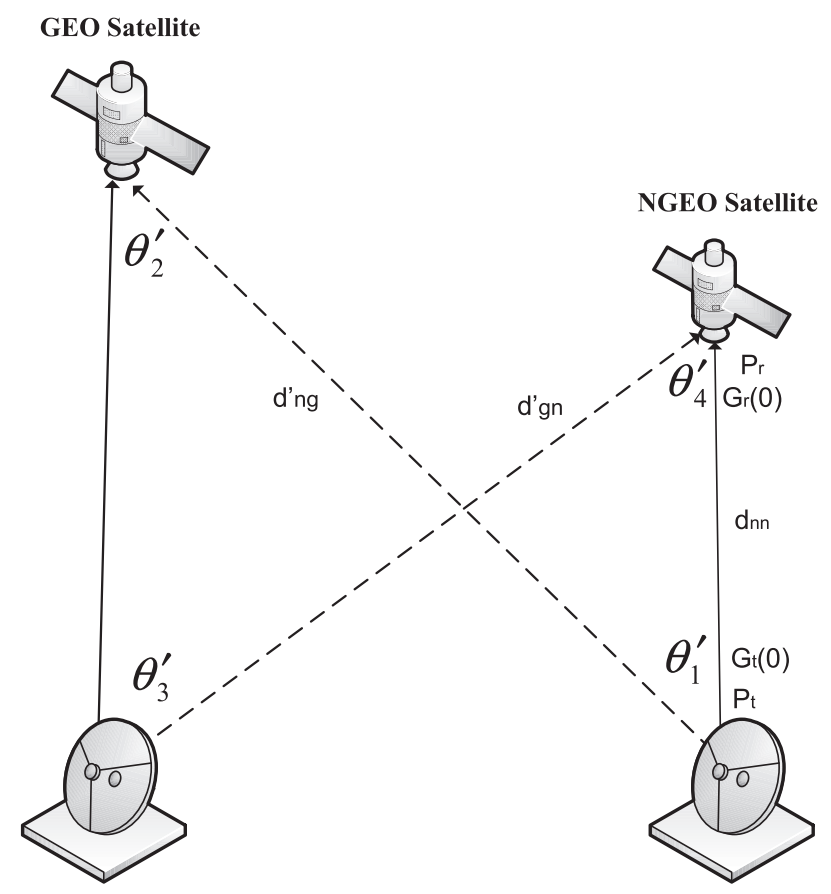

Figure 4. Desired and interference links in the uplink coexistence of GEO and NGEO satellite networks. 


\subsection{Uplink coexistence analysis}

In this scenario, we consider the coexistence of GEO and NGEO links with both operating in normal return mode as shown in Figure 4. There exist the following two interference links: (i) from NGEO earth station to the GEO satellite and (ii) from GEO earth station to the NGEO satellite.

\subsubsection{Problem statement. We study the following problems under this scenario.}

- Since the GEO system is already deployed system and should be protected from the interference caused by the reuse of its operating frequencies, we consider the interfering link between the NGEO earth station to the GEO satellite. In this context, the interference from the NGEO earth station towards the GEO satellite should be below the interference constraint of the GEO satellite.

- When the NGEO link is operating in the spectrum used by the GEO satellite, the NGEO link should provide sufficient QoS to its users while guaranteeing the primary link protection.

3.2.2. Proposed power control in the uplink. We formulate the following feasibility problem under this scenario.

$$
\max _{P} R= \begin{cases}\log _{2}\left(1+\frac{C}{I_{\text {ngeo }}+N}\right), & \frac{C}{I_{n g e o}+N} \geqslant\left(C_{0} / N_{0}\right)_{\text {rns }} \\ 0, & \frac{C}{I_{\text {ngeo }}+N}<\left(C_{0} / N_{0}\right)_{\text {rns }}\end{cases}
$$

subject to $I_{g e o} \leqslant I_{t h}$,

where the expression for $\frac{C}{I_{n g e o}+N}$, that is, uplink SINR at the NGEO satellite considering interference from the GEO earth station, can be written as

$$
S I N R_{u}=\frac{P_{r n s}}{I_{n g e o}+K T_{r n s} W}=\frac{P_{t n e} G_{t n e}(0) G_{r n s}(0)\left(\frac{\lambda}{4 \pi d_{n n}}\right)^{2}}{P_{t g e} G_{t g e}\left(\theta_{3}^{\prime}\right) G_{r n s}\left(\theta_{4}^{\prime}\right)\left(\frac{\lambda}{4 \pi d_{g n}^{\prime}}\right)^{2}+K T_{r n s} W},
$$

where $I_{\text {ngeo }}$ denotes the interference from the GEO earth station towards the NGEO satellite, $P_{r n s}$ denotes the received power at the NGEO satellite, $P_{\text {tne }}$ denotes the power transmitted by the NGEO earth station, $\theta_{3}^{\prime}$ is the offset angle of the GEO earth station transmitting antenna in the direction of the NGEO satellite and $\theta_{4}^{\prime}$ is the offset angle of the NGEO satellite receiving antenna in the direction of the GEO earth station.

It can be noted that if the link budget is not enough to close the link, the terminal cannot transmit anything and does not achieve any rate. More specifically, if $S I N R_{u}<\left(C_{0} / N_{0}\right)_{r n s}$, the signal received at the NGEO satellite is not sufficient to close the link budget. In this case, although the terminal transmits some power, there is no achievable rate and the resource is wasted. In this case, it is better to switch the terminal transmission or switch the transmission to other NGEO satellites that have better link conditions.

In the above problem, first, the feasibility is analyzed based on whether the condition $S I N R_{u} \geqslant$ $\left(C_{0} / N_{0}\right)_{r n s}$ is fulfilled or not. If this condition is satisfied, then the problem can be considered to be feasible; otherwise, this problem becomes infeasible. This feasibility condition can be checked by carrying out the link budget analysis of the interfering link between the NGEO earth station to the GEO satellite. If the problem becomes feasible, the feasibility checking problem in (8) can be formulated into the following optimization problem.

$$
\begin{gathered}
\max _{P} \log _{2}\left(1+\frac{C}{I_{\text {ngeo }}+N}\right) \\
\text { subject to } I_{\text {geo }} \leqslant I_{t h},
\end{gathered}
$$

where $I_{\text {geo }}$ denotes the interference from the NGEO earth station towards the GEO satellite and is given by

$$
I_{g e o}=P_{t n e} G_{t n e}\left(\theta_{1}^{\prime}\right) G_{r g s}\left(\theta_{2}^{\prime}\right)\left(\frac{\lambda}{4 \pi d_{n g}^{\prime}}\right)^{2},
$$


where $P_{\text {tne }}$ denotes the transmitted power of the NGEO earth station, $G_{t n e}\left(\theta_{1}^{\prime}\right)$ denotes the gain of the transmit antenna of the NGEO earth station in the direction of $\theta_{1}^{\prime}, G_{r g s}\left(\theta_{2}^{\prime}\right)$ denotes the gain of the receive antenna of the GEO satellite and $d_{n g}^{\prime}$ denotes the distance between the NGEO earth station and the GEO satellite.

\section{NUMERICAL RESULTS}

In this section, we first present results for the antenna radiation patterns by referring to related ITU-R radio regulations. Then we present numerical results for the proposed power control technique for both uplink and downlink coexistence scenarios considering flat-earth approximation. ${ }^{\S}$ In our numerical results, we consider the worst-case interference scenario by considering both the GEO and NGEO earth stations in the equatorial plane. Furthermore, we assume $90^{\circ}$ elevation angle for the GEO earth station antenna and $5^{\circ}$ angular separation between GEO and NGEO earth stations with respect to GEO satellite. This angular separation approximately corresponds to $3.13 \mathrm{~km}$ distance on the surface of the earth. Subsequently, we calculate the off-axis angles required for calculating beam gains by analyzing the geometry of the considered coexistence scenarios. The link budget parameters for the Ka band GEO satellite and the MEO satellite considering the case of O3b satellite are presented in Tables II and III, respectively.

\subsection{Antenna radiation patterns}

According to ITU-R S.1528, the reference pattern for an NGEO satellite antenna having antenna aperture diameter to wavelength ratio $(D / \lambda<35)$ is given by

$$
G(\theta)=\left\{\begin{array}{l}
G_{m}-3\left(\theta / \theta_{b}\right)^{2} \mathrm{dBi} \text { for } \theta_{b}<\theta<Y \\
G_{m}+L_{s}-25 \log (\theta / Y) \mathrm{dBi} \text { for } Y<\theta<Z \\
L_{F} \mathrm{dBi} \text { for } Z<\theta<180^{\circ}
\end{array}\right.
$$

where $Z=Y \times 10^{0.04\left(G_{m}+L_{s}-L_{F}\right)}, L_{s}$ is the main beam and near-in side-lobe mask cross point (dB) below the peak gain, $L_{F}$ is the far-out side-lobe level $(\mathrm{dBi})$ and $G_{m}=20 \log (D / \lambda)$ represents the maximum gain in the main lobe (dBi). For MEO satellite, $L_{s}=-12$ and $Y=2 \theta_{b}, 2 \theta_{b}$ being the half power beamwidth. The value of $L_{F}$ is $0 \mathrm{dBi}$ for ideal patterns.

According to the recommendation ITU-R S.1428-1, when there are multiple interfering sources whose positions vary substantially with time, the level of interference received inevitably depends on the troughs as well as the peaks in the antenna side lobe gain pattern of the victim or source of interference, respectively. In this context, the ITU-R S.1428 recommends the following reference earth station pattern for both GEO and NGEO links (for antennas having $D / \lambda>100$ ).

$$
G(\theta)=\left\{\begin{array}{l}
G_{\max }-2.5 \times 10^{-3}\left(\frac{D}{\lambda} \theta\right)^{2} \mathrm{dBi} \text { for } 0<\theta<\theta_{m} \\
G_{1} \text { for } \theta_{m} \leqslant \theta<\left(95 \frac{\lambda}{D}\right) \\
29-25 \log (\theta) \text { for } \theta_{r} \leqslant \theta<10^{\circ} \\
34-30 \log (\theta) \text { for } 10^{\circ} \leqslant \theta<34.1^{\circ} \\
-12 \mathrm{dBi} \text { for } 34.1^{\circ} \leqslant \theta<80^{\circ} \\
-7 \mathrm{dBi} \text { for } 80^{\circ} \leqslant \theta<120^{\circ}
\end{array}\right.
$$

where $G_{\max }=20 \log (D / \lambda)+8.4 \mathrm{dBi}, \quad G_{1}=-1+15 \log (D / \lambda) \mathrm{dBi}, \quad \theta_{m}=\frac{20 \lambda}{D} \sqrt{G_{\max }-G_{1}} \mathrm{deg}$, $\theta_{r}=15.85(D / \lambda)^{-0.6} \mathrm{deg}$.

\footnotetext{
$\S$ The exact analysis of interference between GEO and NGEO networks requires a 3D model, and in this paper, we consider the flat-earth approximation for the sake of simplicity. However, the proposed techniques are easily applicable for the real practical scenarios as well.
} 
Furthermore, the recommendation ITU-R S.672-4 provides antenna patterns for the GEO satellite given by

$$
G(\theta)=\left\{\begin{array}{l}
G_{m}-3\left(\theta / \theta_{b}\right)^{2} \mathrm{dBi} \text { for } \theta_{b}<\theta<a \theta_{0} \\
G_{m}+L_{s} \mathrm{dBi} \text { for } a \theta_{0}<\theta<b \theta_{0} \\
G_{m}+L_{s}+20-25 \log 10\left(\theta / \theta_{0}\right) \mathrm{dBi} \text { for } b \theta_{0}<\theta<\theta_{1} \\
0, \text { for } \theta_{1} \leqslant \theta<90^{\circ} \\
3, \text { for } 90^{\circ} \leqslant \theta<180^{\circ},
\end{array}\right.
$$

where $G_{m}$ is the maximum gain in the main lobe $(\mathrm{dBi}), \theta_{0}$ is the one half the $3 \mathrm{~dB}$ beamwidth in the plane of interest, $\theta_{1}$ is the value of the $\theta$ when the $G(\theta)=G_{m}+L_{s}+20-25 \log 10\left(\theta / \theta_{0}\right)$ becomes equal to $0 \mathrm{~dB}, L_{s}$ is the desired side-lobe level relative to peak gain, and $(a, b)$ are numeric values and vary based on the value of $L_{s}$. For $L_{s}=-20 \mathrm{~dB}$, the values of $a$ and $b$ are 2.58 and 6.32, respectively.

For our simulation purpose, we analyze the gain patterns of GEO/NGEO earth station terminals and the GEO/NGEO satellites using relevant ITU-R recommendations. Figure 5 shows the gain pattern of GEO/NGEO earth station antenna for transmission purpose (i.e., in the uplink direction) (carrier frequency $=28.28 \mathrm{GHz}$ ), and Figure 6 presents the gain pattern of GEO/NGEO earth station antenna for reception purpose (carrier frequency $=18.48 \mathrm{GHz}$ ) using ITU-R S.1428. Similarly, Figure 7 presents

Table II. Link budget parameters for a Ka-band GEO satellite.

\begin{tabular}{|c|c|}
\hline Parameter & Value \\
\hline \multicolumn{2}{|l|}{ Parameters for satellite } \\
\hline Orbit & GEO (circular, equatorial) \\
\hline Satellite height & $35,786 \mathrm{~km}$ \\
\hline Round trip delay & $500 \mathrm{~ms}$ \\
\hline Satellite noise temperature & $575^{\circ} \mathrm{K}$ \\
\hline Max antenna Tx gain & $52 \mathrm{dBi}$ \\
\hline Max antenna Rx gain & $55.5 \mathrm{dBi}$ \\
\hline TWTA RF power@ saturation $P_{\mathrm{pt}}$ & $80 \mathrm{~W}$ \\
\hline $\mathrm{OBO}$ & $5 \mathrm{~dB}$ \\
\hline Satellite EIRP & $71 \mathrm{dBW}$ \\
\hline Polarization & Single \\
\hline Antenna efficiency & $75 \%$ \\
\hline Reflector size & $2.2 \mathrm{~m}$ \\
\hline $3 \mathrm{~dB}$ beamwidth & $0.82^{\circ}$ \\
\hline \multicolumn{2}{|l|}{ Parameters for gateway } \\
\hline Uplink carrier frequency & $28.28 \mathrm{GHz}$ \\
\hline Gateway antenna diameter & $8 \mathrm{~m}$ \\
\hline Gateway antenna efficiency & $60 \%$ \\
\hline Max gateway antenna gain & $65.8 \mathrm{dBi}$ \\
\hline HPA peak output power & $250 \mathrm{~W}$ \\
\hline Gateway EIRP & $66 \mathrm{dBW}$ \\
\hline uplink free space loss & $212.5 \mathrm{~dB}$ \\
\hline Gateway bandwidth & $1 \mathrm{GHz}$ \\
\hline Uplink C/N (clear sky) & $38.7 \mathrm{~dB}$ \\
\hline Atmospheric fade margin (@ 99.9\% availability) & $14 \mathrm{~dB}$ \\
\hline Uplink C/N & $24.7 \mathrm{~dB}$ \\
\hline \multicolumn{2}{|l|}{ Parameters for user terminal } \\
\hline Downlink carrier frequency & $18.48 \mathrm{GHz}$ \\
\hline User link availability & $99.7 \%$ \\
\hline Terminal antenna diameter & $0.75 \mathrm{~m}$ \\
\hline Terminal antenna efficiency & $60 \%$ \\
\hline Downlink free space loss & $209 \mathrm{~dB}$ \\
\hline User link bandwidth & $500 \mathrm{MHz}$ \\
\hline Clear sky receiver temperature & $207^{\circ} \mathrm{K}$ \\
\hline Bandwidth per Beam & $125 \mathrm{MHz}$ \\
\hline Carriers per beam & 1 \\
\hline Bandwidth per carrier & $125 \mathrm{MHz}$ \\
\hline Downlink C/I (total interference) & $16 \mathrm{~dB}$ \\
\hline Fade margin (@ 99.7\% availability) & $6 \mathrm{~dB}$ \\
\hline Downlink $\mathrm{C} / \mathrm{N}$ & $16.1 \mathrm{~dB}$ \\
\hline Downlink $\mathrm{C} /(\mathrm{N}+\mathrm{I})$ & $14 \mathrm{~dB}$ \\
\hline Total $\mathrm{C} /(\mathrm{N}+\mathrm{I})$ & $13.6 \mathrm{~dB}$ \\
\hline $\mathrm{C} / \mathrm{N}$ range & {$[0,20] \mathrm{dB}$} \\
\hline Terminal Tx power for RL & $1 \mathrm{~W}$ \\
\hline
\end{tabular}


Table III. Link budget parameters for MEO satellite (O3b).

\begin{tabular}{|c|c|}
\hline Parameter & Value \\
\hline \multicolumn{2}{|l|}{ Parameters for satellite } \\
\hline Orbit & MEO (circular, equatorial) \\
\hline Satellite height & $8062 \mathrm{~km}$ \\
\hline Trunking capacity & $1.2 \mathrm{Gbps}$ per beam \\
\hline Constellation size & 8 \\
\hline Orbit spacing & $45^{\circ}$ \\
\hline Orbital inclination & $<0.1^{\circ}$ \\
\hline Round trip delay & $120 \mathrm{~ms}$ \\
\hline Number of contacts per day & 4 per day \\
\hline Polarization & Dual orthogonalization \\
\hline Beam diameter & $700 \mathrm{~km}$ \\
\hline Transponder bandwidth & $2 \times 216 \mathrm{MHz}$ per beam \\
\hline Channel bandwidth & $216 \mathrm{MHz}$ \\
\hline Spacing between satellites & $45^{\circ}$ \\
\hline Tx antenna gain & $31.54 \mathrm{dBi}$ \\
\hline Antenna diameter & $0.3616 \mathrm{~m}$ \\
\hline Half power beamwidth & $3.2^{\circ}$ \\
\hline OBO & $-3.8 \mathrm{~dB}$ \\
\hline EIRP per channel per carrier & $44.37 \mathrm{dBW}$ \\
\hline \multicolumn{2}{|l|}{ Parameters for gateway } \\
\hline Uplink carrier frequency & Ka band $(28.28 \mathrm{GHz})$ \\
\hline Gateway beams & 2 per satellite \\
\hline Tx channels per HPA & 5 \\
\hline uplink free space loss & $200.5 \mathrm{~dB}$ \\
\hline Tx antenna gain & $64.90 \mathrm{~dB}$ \\
\hline Tx EIRP per channel & $78.66 \mathrm{dBW}$ \\
\hline Fade margin (@ 99.5\% availability) & $8.06 \mathrm{~dB}$ \\
\hline Uplink C/N & $11.51 \mathrm{~dB}$ \\
\hline \multicolumn{2}{|l|}{ Parameters for user terminal } \\
\hline Downlink carrier frequency & Ka band $(18.48 \mathrm{GHz})$ \\
\hline Number of user beams & 10 per satellite \\
\hline Downlink free space loss & $197 \mathrm{~dB}$ \\
\hline $\mathrm{Rx}$ antenan gain $(\mathrm{dBi})$ & 56.13 \\
\hline Antenna diameter & $2.4 \mathrm{~m}$ \\
\hline Rx effective $\mathrm{G} / \mathrm{T}$ & $30.3 \mathrm{~dB} / \mathrm{K}$ \\
\hline Rx power per channel & $-105.14 \mathrm{dBW}$ \\
\hline Rx flux density per channel & $-114.48 \mathrm{dBW} / \mathrm{m}^{2}$ \\
\hline Downlink $\mathrm{C} / \mathrm{N}$ & $14.81 \mathrm{~dB}$ \\
\hline
\end{tabular}

the gain pattern of the GEO satellite antenna using recommendation ITU-R S.672-4, and Figure 8 presents the gain pattern of the NGEO satellite using recommendation ITU-R S.1528.

\subsection{Uplink analysis}

We consider the coexistence of a GEO and an NGEO link, both operating in the normal return mode. As mentioned in the earlier section, we tackle the problem of link feasibility analysis and adaptive power control to maximize the rate of the NGEO link. To check whether the NGEO link is feasible or not, we plot the SINR received at the NGEO satellite considering the desired transmission from the NGEO earth station terminal and the interfering transmission from the GEO earth station terminal.

Figure 9 shows the transmit power of the NGEO earth station versus off-axis angle. The values of transmit power for different off-axis angles were obtained by solving the optimization problem given by (10). As shown in the figure legends, the values of interference threshold, that is, $I_{t h}$, was considered to be -150 and $-170 \mathrm{dBW}$. From the figure, it can be noted that when the in-line event occurs, that is, when the NGEO earth station is in-line with the GEO and NGEO satellites, the transmit power of the 
NGEO station should be decreased and it can be increased as we go away from the boresight direction in order to maximize the rate of the secondary link.

Figure 10 shows the SINR versus off-axis angle for the considered uplink coexistence scenario. This variation in the SINR comes from the fact that the NGEO satellite is moving over time, and the interfering signal received by it depends on the angular position with respect to the beampattern of the GEO earth station terminal. In this context, the beampatterns given by Figures 6 and 8 are used for modeling the gains of the GEO earth station and the NGEO satellite, respectively. It should be noted that the value of SINR is the lowest when the GEO earth station terminal falls in the in-line position of the NGEO satellite. Let us consider the minimum required value of SINR, that is, SINR $_{\min }$, to close the NGEO link as $6 \mathrm{~dB} .{ }^{\pi}$ From the figure (Figure 10), it can be noted that for the interference threshold value of $I_{t h}=-170 \mathrm{dBW}$, the SINR received at the NGEO link is not sufficient to close the link in the range between $\pm 2.5^{\circ}$ of the maximum gain position and hence the problem in (8) is not feasible within this angular region. Beyond this region, the SINR is sufficient to close the NGEO link and the

\footnotetext{
${ }^{\top}$ In practice, more precise value of $\mathrm{SINR}_{\min }$ can be obtained from standards or the regulatory constraints for a particular NGEO satellite system
}

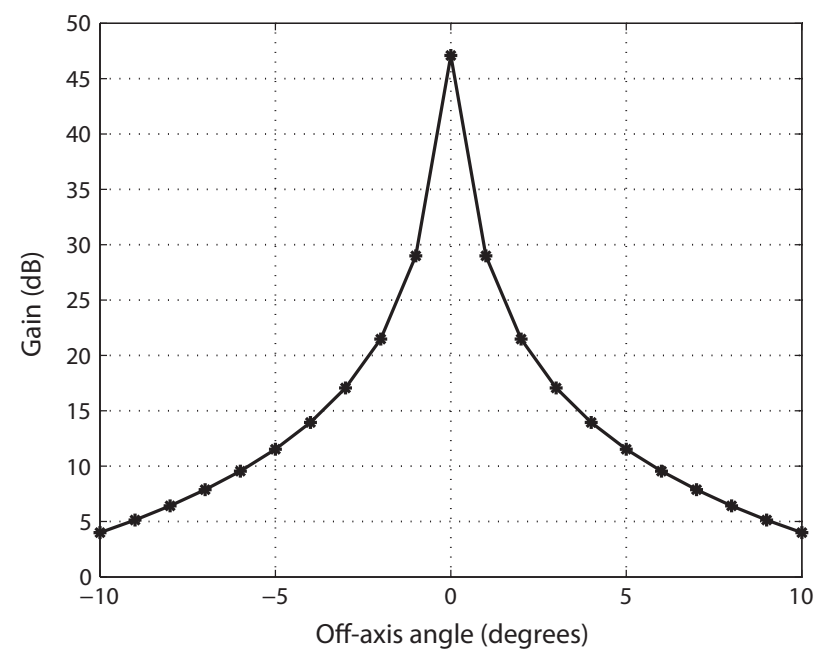

Figure 5. Gain pattern of earth station antenna (GEO/NGEO) in the uplink using ITU-R S.1428.

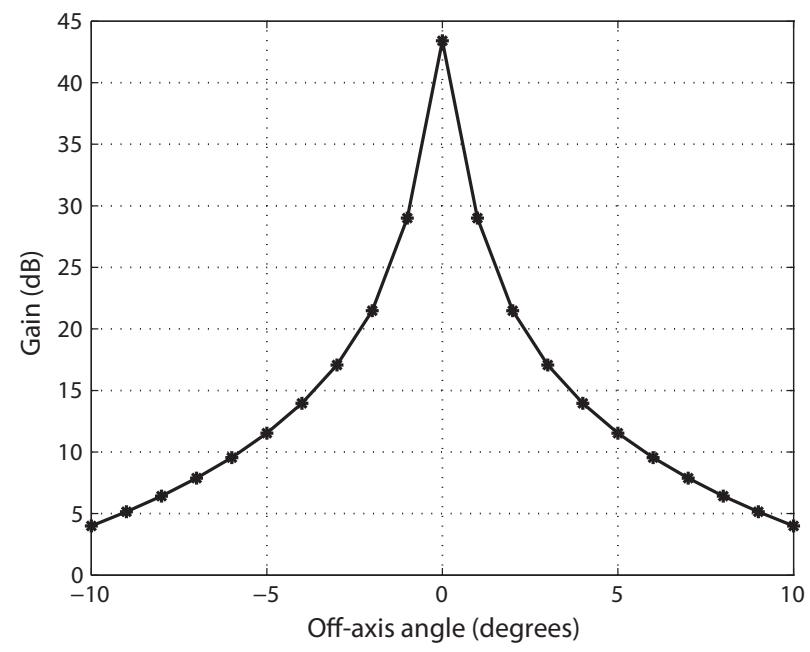

Figure 6. Gain pattern of earth station antenna (GEO/NGEO) in the downlink using ITU-R S.1428. 
IN-LINE INTERFERENCE MITIGATION TECHNIQUES

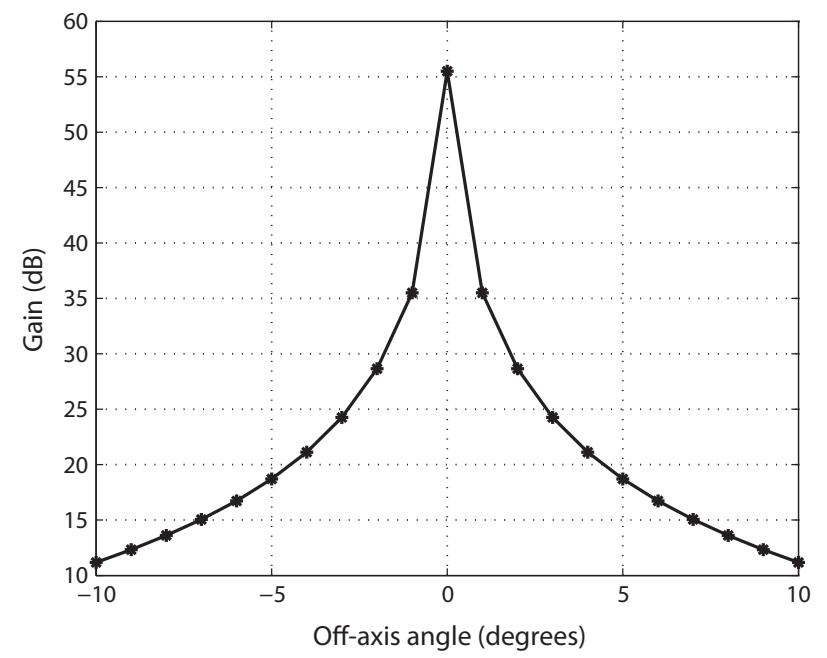

Figure 7. Gain pattern of GEO satellite receive antenna using ITU-R S.672-4.

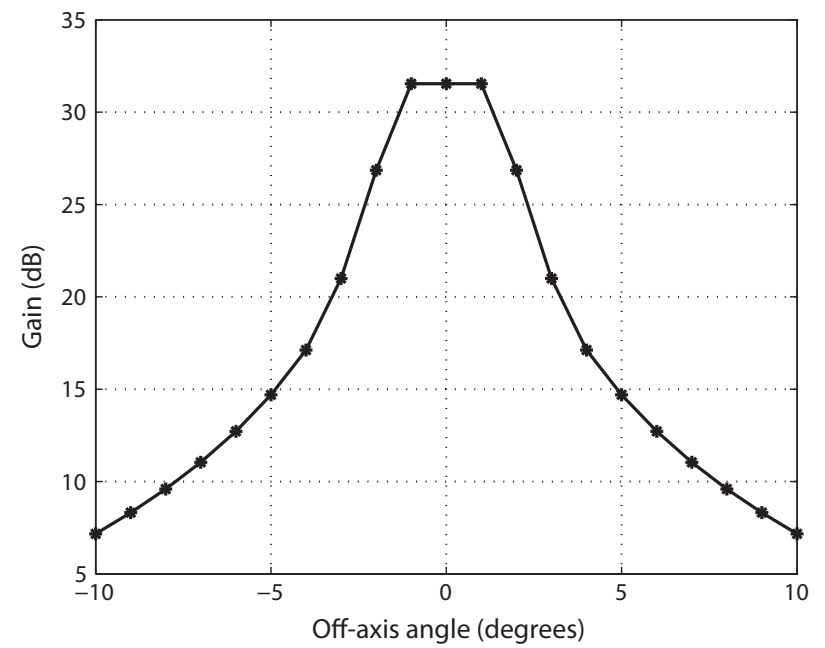

Figure 8. Gain pattern of NGEO satellite antenna using ITU-R S.1528.

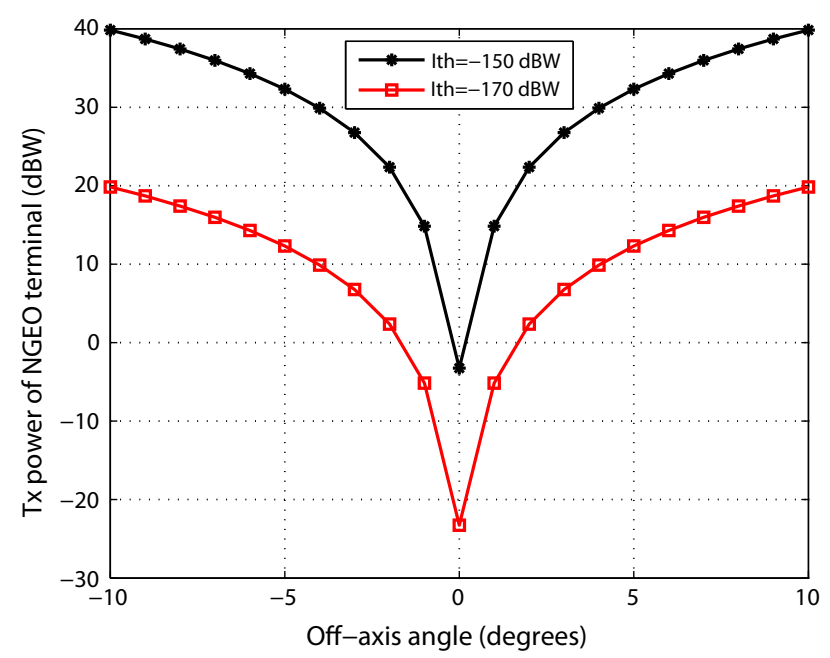

Figure 9. Transmit power of the NGEO earth station terminal for the uplink coexistence scenario of GEO and NGEO links. 


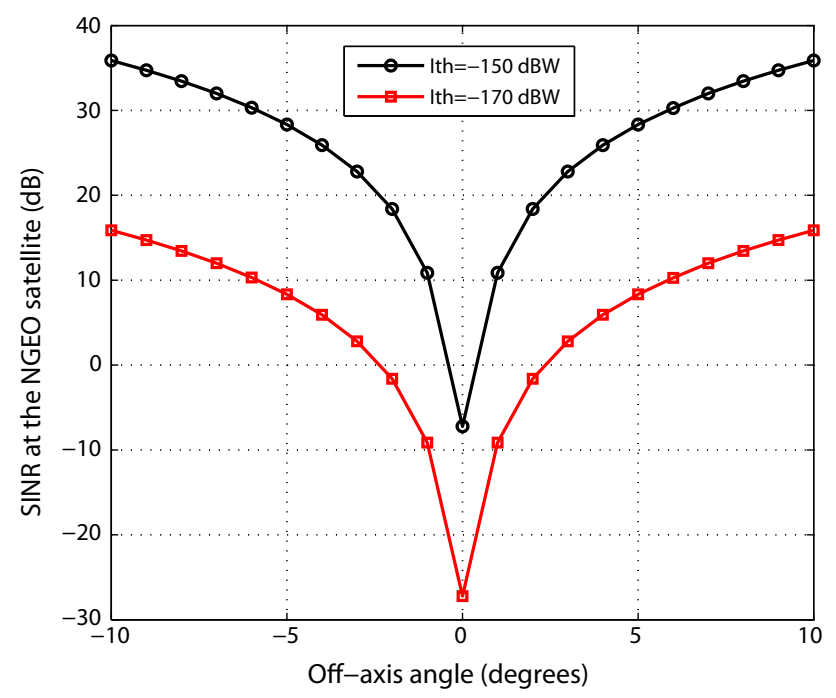

Figure 10. SINR at the NGEO satellite for the uplink coexistence scenario of GEO and NGEO links.

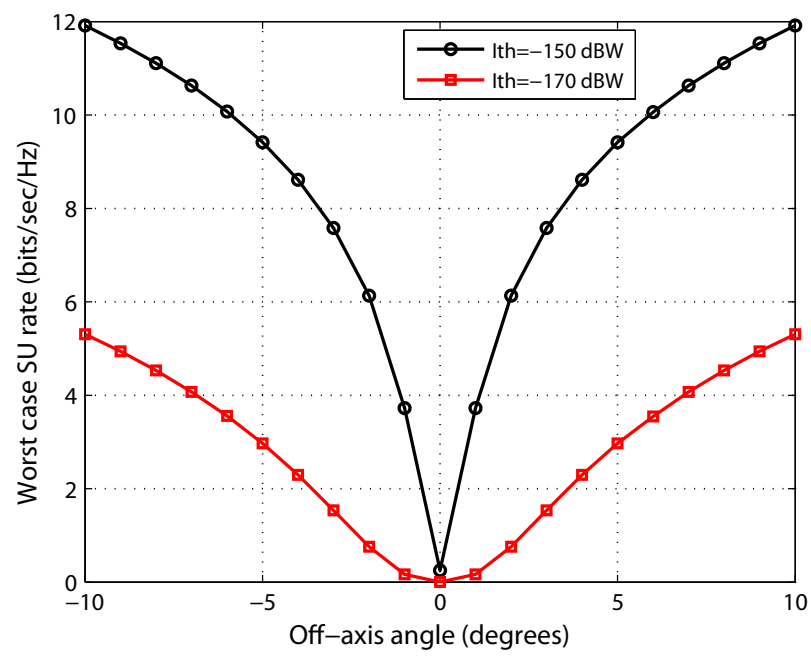

Figure 11. SU rate for the uplink coexistence scenario of GEO and NGEO links.

feasibility check problem in (8) reduces to the optimization problem in (10). It should be noted that this feasible range depends on the allowable interference threshold at the GEO satellite. Furthermore, we present the plot of sum-rate of the NGEO link versus off-axis angle in Figure 11. From the figure, it can be noted that the achievable sum-rate near to the boresight direction is very low, and it increases as the off-axis angle is increased. The SU rate (SR) plotted in Figure 11 is obtained from the SINR plot in Figure 10 using the relation $\mathrm{SR}=\log _{2}\left(1+10^{0.1 \mathrm{SINR}}\right)$.

\subsection{Downlink analysis}

For the downlink coexistence scenario, we solve the optimization problem given by (6). Figure 12 shows the SINR versus off-axis angles for the downlink coexistence scenario. In these simulation results, the values of interference threshold and the desired carrier power were considered to be -150 and $-105 \mathrm{dBW}$, respectively. The optimum value of power was found to be $12.2747 \mathrm{dBW}$. The NGEO satellite was considered at an angular distance of $5^{\circ}$ from the boresight direction $\left(0^{\circ}\right)$ of the main beam of the GEO satellite. Figure 13 presents the worst-case SU rate versus off-axis angle for the downlink coexistence scenario. It can be noted that the worst-case SU rate slightly increases as we move away 


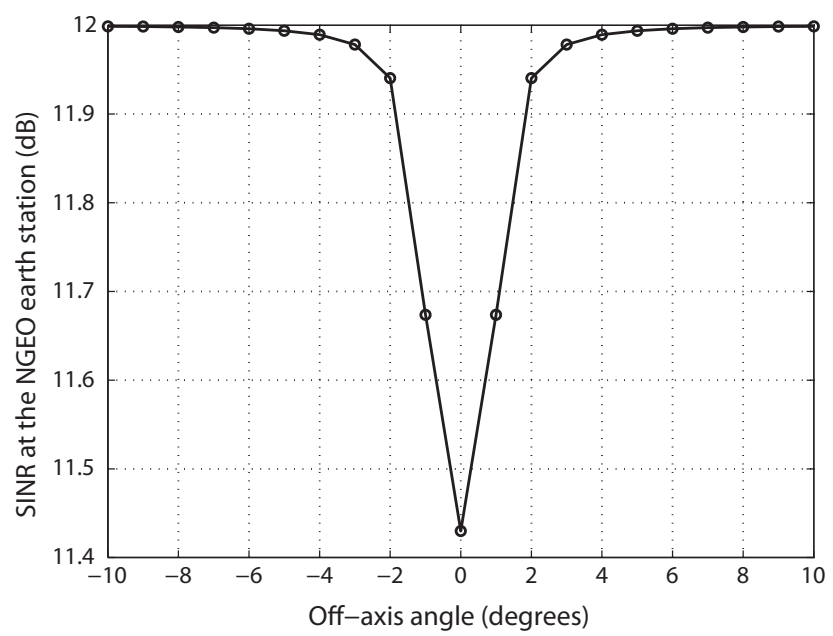

Figure 12. SINR at the NGEO earth station for the downlink coexistence scenario of GEO and NGEO links $\left(I_{t h}=-150 \mathrm{dBW}, C_{0}=-105 \mathrm{dBW}\right)$.

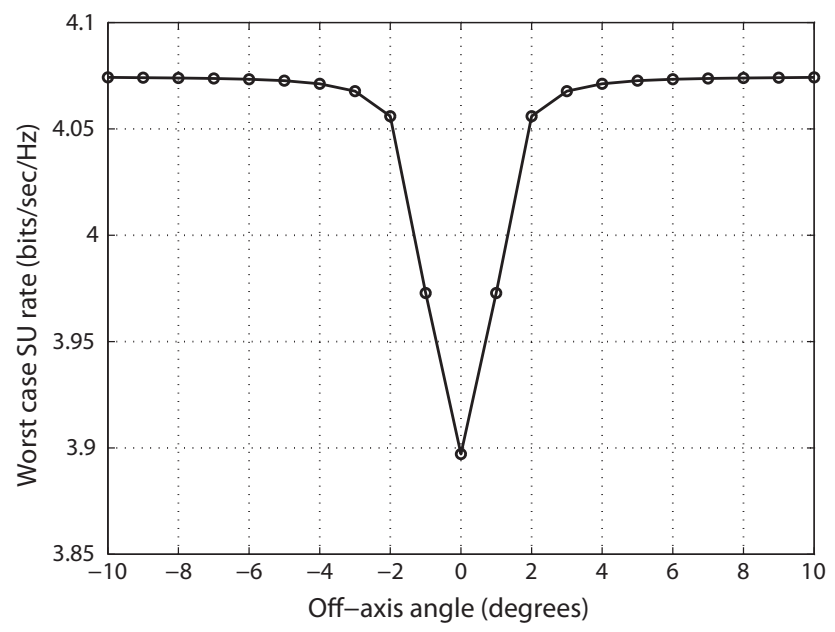

Figure 13. SU rate for the downlink coexistence scenario of GEO and NGEO links $\left(I_{t h}=-150 \mathrm{dBW}, C_{0}=\right.$ $-105 \mathrm{dBW})$.

from the boresight direction and remains more or less constant beyond $5^{\circ}$. The SU rate (SR) plotted in Figure 13 is obtained from the SINR plot in Figure 12 using the relation $\mathrm{SR}=\log _{2}\left(1+10^{0.1 \mathrm{SINR}}\right)$.

In order to show the effect of desired carrier threshold value $C_{0}$ on the transmit power as well as on the SU rate, we plot transmit power versus carrier threshold in Figure 14 and SU rate versus carrier threshold in Figure 15. From Figure 14, it can be noted that as the desired carrier threshold for the NGEO earth station increases, the required transmit power also increases. This experiment was carried out by setting the value of interference threshold towards the GEO earth station as $-150 \mathrm{dBW}$. Different levels of carrier threshold may be required at the NGEO terminal depending on the type of modulation schemes implemented. For example, a Binary Phase Shift Keying (BPSK) scheme requires smaller value of carrier threshold and the required minimum value of carrier threshold increases for higher modulation schemes. The conclusion from Figures 14 and 15 is that the transmit power at the NGEO satellite can be adjusted in order to provide the desired values of carrier power as well as the user rate by guaranteeing the sufficient protection of the GEO earth station. 


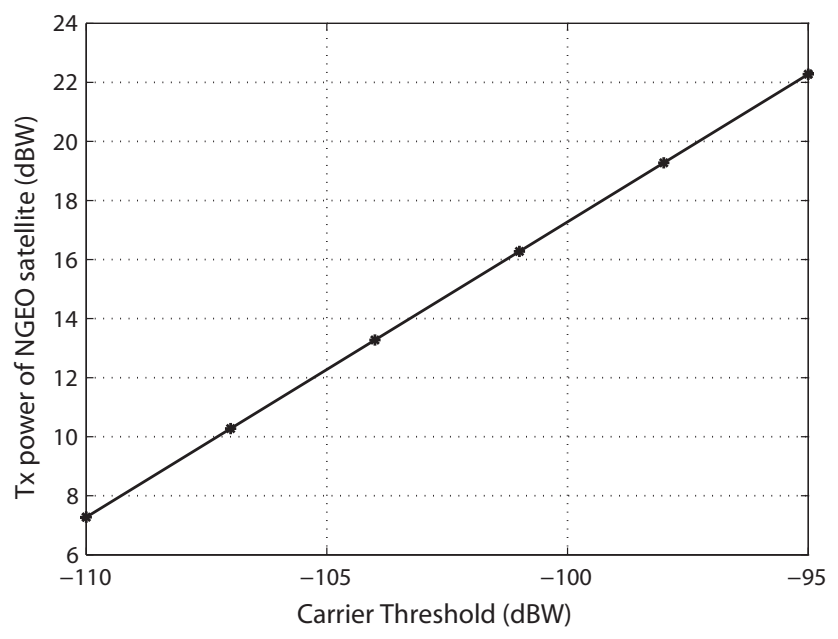

Figure 14. Transmit power versus carrier threshold for the downlink coexistence scenario of GEO and NGEO links $\left(I_{t h}=-150 \mathrm{dBW}\right)$.

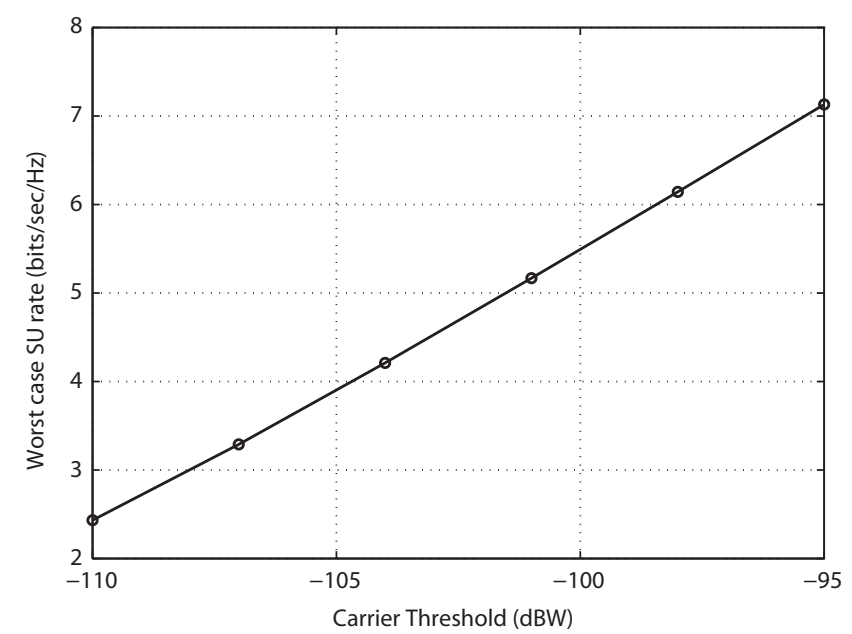

Figure 15. SU rate versus carrier threshold for the uplink coexistence scenario of GEO and NGEO links $\left(I_{t h}=\right.$ $-150 \mathrm{dBW})$.

\subsection{NGEO capacity}

In this section, we evaluate the total capacity of an NGEO satellite using the proposed power controlbased method and compare with the following capacities: (i) the NGEO only capacity (without GEO presence) and (ii) the NGEO capacity achieved by using ITU-R angular discrimination-based approach. For the evaluation of NGEO total capacity, we use the analytical method proposed in [4] in order to take account of the statistical behavior of the interference events. For the simplicity of analysis, we consider only one NGEO satellite in an orbit of height $8062 \mathrm{~km}$ with its plane inclined at $20^{\circ}$ to the equatorial plane.

As mentioned before, ITU-R S.1325 suggests different methodologies in order to avoid interference with the GEO satellite when the in-line situation occurs. Out of these approaches, we consider the discrimination angle-based approach. In this approach, whenever an NGEO satellite terminal sees an angular separation of less than $\pm \alpha^{\circ}$ between GEO and NGEO satellites, it switches off its transmission. On the other hand, in our proposed power control-based approach, the NGEO satellite terminal can transmit with the controlled power, determined based on the interference threshold of the GEO satellite, even if the NGEO satellite enters into the region of discrimination zone.

Let $(\theta, \phi)$ denote the location of an NGEO satellite at a certain time instant with $\theta$ being latitude and $\phi$ being the corresponding longitude. This can be considered as a reference satellite position of an 
NGEO network while considering multiple NGEO satellites as in [4]. Since the NGEO satellite moves over the time, the values of $\theta$ and $\Phi$ vary over the time. Depending on the inclination of the NGEO satellite's plane with respect to the equatorial plane and the coverage region of interest on the surface of the earth, the NGEO satellite appears over a certain grid (in terms of the latitude and longitude) with a certain probability. In the coexistence case considered in this paper, the second network is a GEO network and the position of the GEO satellite remains stationary with respect to a terminal located on the surface of the earth. While considering the uplink coexistence of these two networks, the desired signal level at the NGEO satellite and the interference signal level at the GEO satellite vary as the position of NGEO satellite changes over the time. This dynamics can be captured in the analysis by considering the probability of an NGEO satellite being in a certain grid $\mathbf{x}=(\theta, \phi)$ position. Let $\gamma$ denote the angle between the NGEO orbital plane and the equatorial plane, then the range of interest becomes $-\gamma \leqslant \theta \leqslant \gamma,-\pi \leqslant \phi \leqslant \pi$ [4]. By modeling $\mathbf{x}$ as a random variable with probability density function (PDF) $P_{\mathbf{x}}(\theta, \phi)$, the desired and interference signal levels can be computed while considering the satellite location probability density function. The expression for $P_{\mathbf{x}}(\theta, \phi)$ is given by [4]

$$
P_{\mathbf{x}}(\theta, \phi)=\left\{\begin{array}{l}
\frac{1}{2 \pi^{2}} \frac{\cos (\theta)}{\sqrt{\sin ^{2}(\gamma)-\sin ^{2}(\theta)}},-\pi \leqslant \phi \leqslant \pi,-\gamma \leqslant \theta \leqslant \gamma \\
0, \text { otherwise. }
\end{array}\right.
$$

It can be noted that the uplink SINR given by (9) and the interference towards the GEO satellite (11) depends on the geometry of the problem since the values of the parameters $\theta_{3}^{\prime}, \theta_{4}^{\prime}, \theta_{1}^{\prime}, \theta_{2}^{\prime}, d_{n n}, d_{n g}^{\prime}$ and $d_{g n}^{\prime}$ vary depending on the position of the NGEO satellite. As illustrated in [4], after calculating the NGEO satellite location density function using (12), the corresponding density function of $S I N R_{u}$ in (9) can be computed.

For simulation of the aforementioned procedure, we consider the SES ASTRA 2D GEO satellite located at $28.2^{\circ}$ East (E) and a GEO satellite terminal with elevation angle of $90^{\circ}$, that is, at the grid position of $28.2^{\circ} \mathrm{E}$ longitude and $0^{\circ}$ latitude. Furthermore, we consider an NGEO satellite at an orbit of height $8062 \mathrm{~km}$ with its plane having $20^{\circ}$ inclination $\|$ to the equatorial plane and an NGEO satellite terminal located at the grid position of $22.2281^{\circ} \mathrm{E}$ in the equatorial plane. Then in order to evaluate the capacity of a NGEO satellite in a specified orbital position, we define the following terms.

(1) Orbital Capacity: It is defined as the capacity for a specific NGEO orbital location.

(2) Average Orbital Capacity: It is the orbital capacity normalized according to the probability of the NGEO orbital location of interest.

(3) Total Capacity: Total capacity integrated over the considered orbital locations.

Table IV provides the simulation parameters used for generating results presented in this subsection. Figure 16 depicts the NGEO orbital capacity versus satellite orbital position without considering the

"The inclination of $\mathrm{O} 3 \mathrm{~b}$ orbit is less than $0.1^{\circ}$, and in this section, we consider the general NGEO case without being specific to the $\mathrm{O} 3 \mathrm{~b}$ case.

Table IV. Simulation parameters used for calculating NGEO total capacity.

\begin{tabular}{lc}
\hline Parameter & Value \\
\hline NGEO earth station transmit power & $12 \mathrm{dBW}$ \\
GEO earth station transmit power & $12 \mathrm{dBW}$ \\
GEO earth station location & $0^{\circ}$ latitude, $28.2^{\circ} \mathrm{E}$ longitude \\
NGEO earth station location & $0^{\circ}$ latitude, $28.2281^{\circ} \mathrm{E}$ longitude \\
GEO satellite location & $0^{\circ}$ latitude, $28.2^{\circ} \mathrm{E}$ longitude \\
GEO satellite antenna pattern & ITU-R S.672-4 \\
NGEO satellite antenna pattern & ITU-R S.1528 \\
GEO earth station antenna pattern & ITU-R S.1528 \\
NGEO station antenna pattern & ITU-R S.1428 \\
NGEO satellite latitude range & $20^{\circ} \mathrm{S}$ to $20^{\circ} \mathrm{N}$ \\
NGEO satellite longitude range & $10^{\circ} \mathrm{E}$ to $45^{\circ} \mathrm{E}$ \\
Discrimination angle for angular discrimination method & $10^{\circ}$ \\
GEO interference threshold for power control approach & $-130 \mathrm{dBW}$ \\
Carrier bandwidth & $500 \mathrm{MHz}$ \\
Noise power & $-117 \mathrm{dBW}$ \\
\hline
\end{tabular}




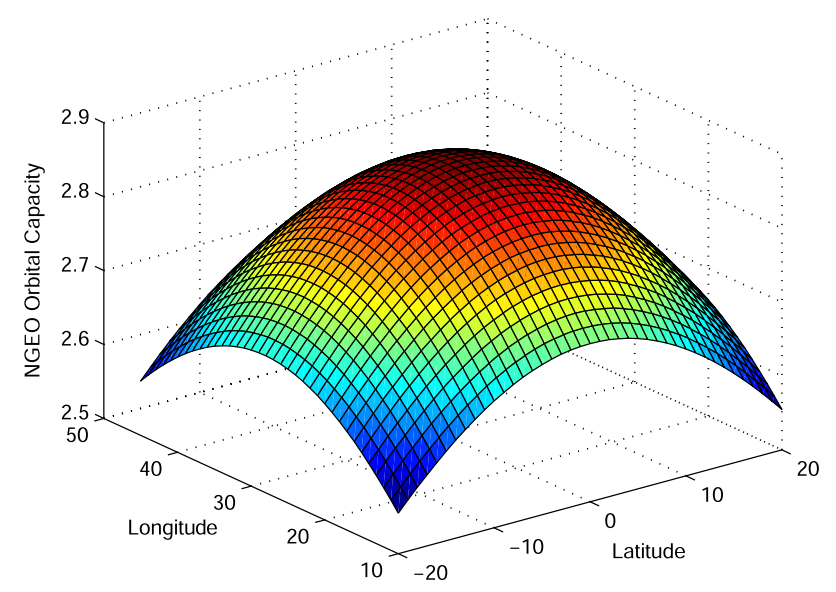

Figure 16. NGEO orbital capacity versus NGEO satellite position without considering the GEO link.

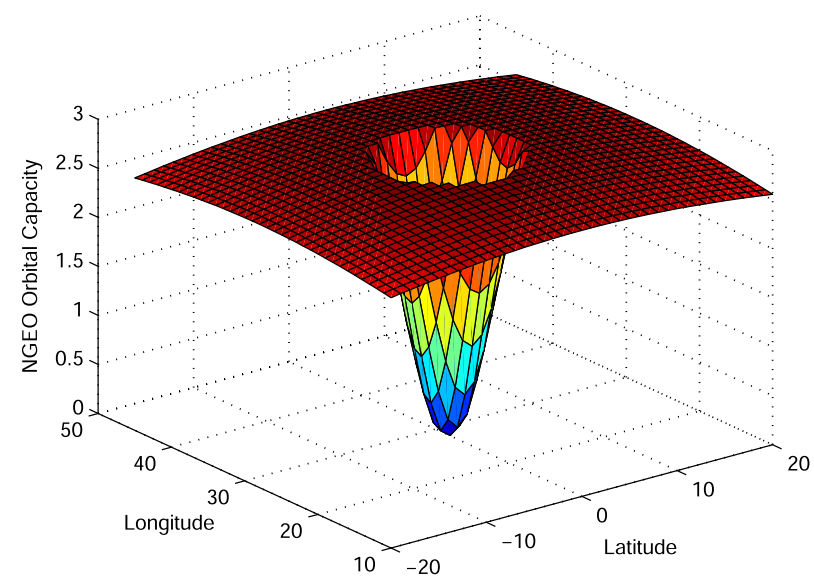

Figure 17. NGEO orbital capacity versus orbital position with power control at the NGEO terminal.

GEO link. As noted in Table IV, we consider the NGEO orbital region of $-20^{\circ} \leqslant \theta \leqslant 20^{\circ}$ and $10^{\circ} \leqslant$ $\phi \leqslant 45^{\circ}$ for evaluating the NGEO capacity. Similarly, Figure 17 depicts the NGEO orbital capacity versus orbital position with the proposed power control-based approach, and Figure 18 presents the NGEO orbital capacity versus orbital position with ITU-R discrimination angle-based approach. In the presented results (Figures 17 and 21), we control the NGEO transmission in such a way that the transmitted power does not exceed the maximum power of the NGEO satellite terminal indicated in Table IV.

Figure 19 depicts the probability density function of NGEO satellite location calculated based on (12). It can be noted the probability of the NGEO satellite being in a particular grid area varies with the latitude of the NGEO satellite with its value being high near to the latitude value equal to the inclination angle of the NGEO satellite. In order to evaluate the average capacity of the NGEO satellite, we apply the PDF plotted in Figure 19 to the NGEO orbital capacities plotted in Figures 16-18. Figure 20 indicates the NGEO only average capacity versus satellite position without considering the GEO presence. Furthermore, Figure 21 depicts the average NGEO capacity versus satellite position with the proposed power control, and Figure 22 presents the average NGEO capacity versus satellite position with the ITU-R discrimination angle-based approach.

Table V provides the comparison of the total NGEO capacity for three different scenarios, which is calculated by integrating the average orbital capacity over the considered orbital locations, that is, $-20^{\circ} \leqslant \theta \leqslant 20^{\circ}$ and $10^{\circ} \leqslant \phi \leqslant 45^{\circ}$. It can be noted that the NGEO total capacity is the highest for 


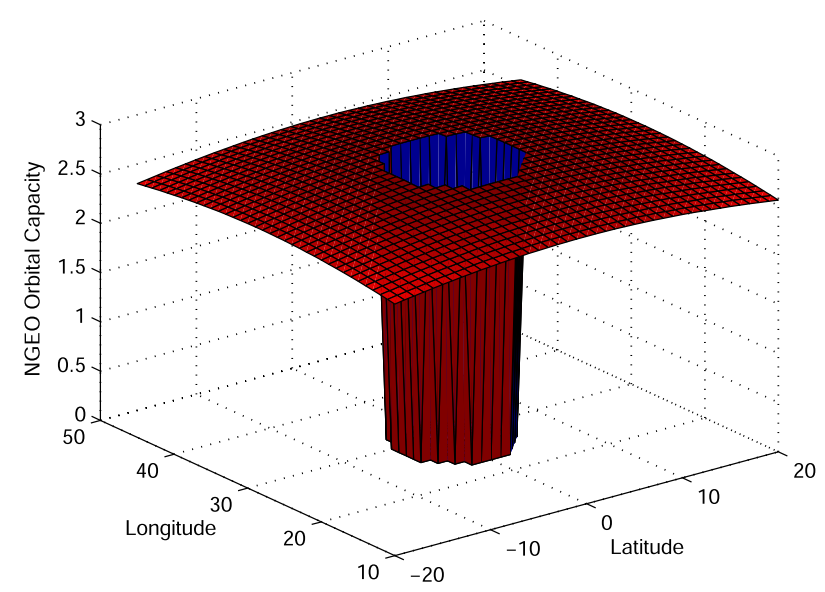

Figure 18. NGEO orbital capacity versus orbital position with ITU-R discrimination angle-based approach.

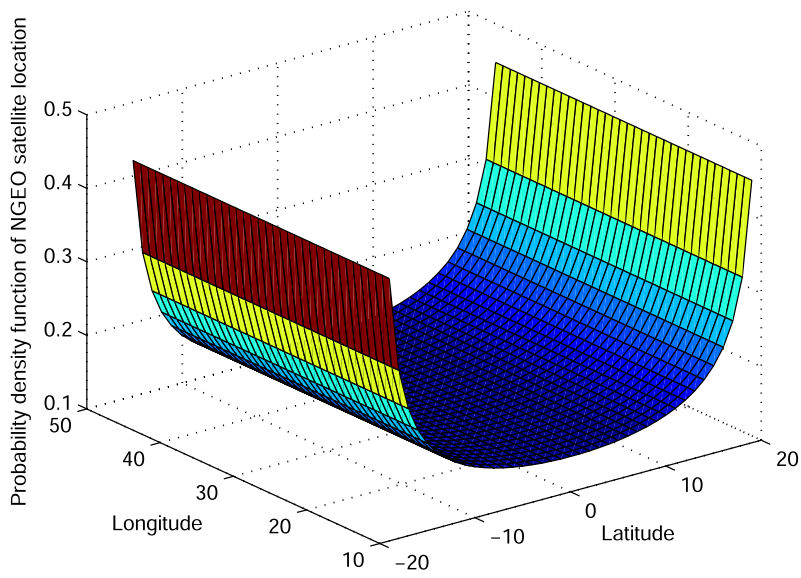

Figure 19. Probability density function of NGEO satellite location.

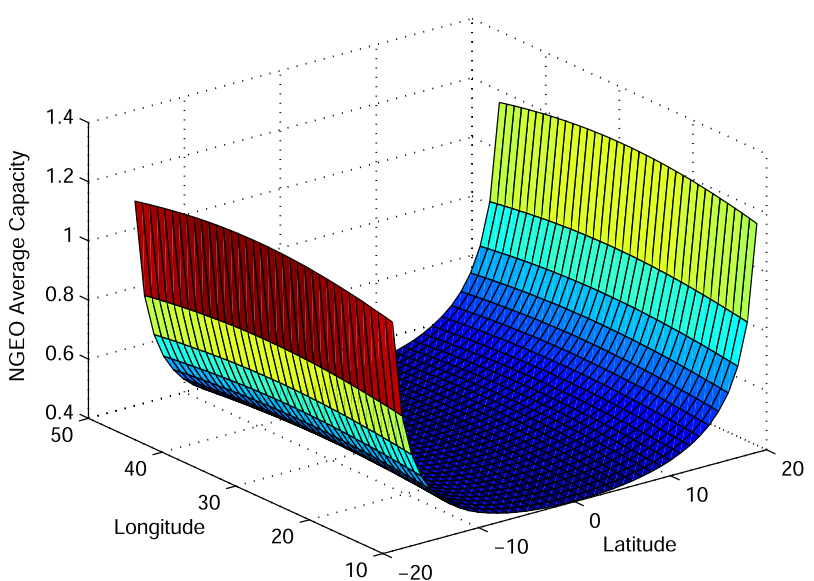

Figure 20. NGEO average capacity versus orbital position without considering the GEO interference. 


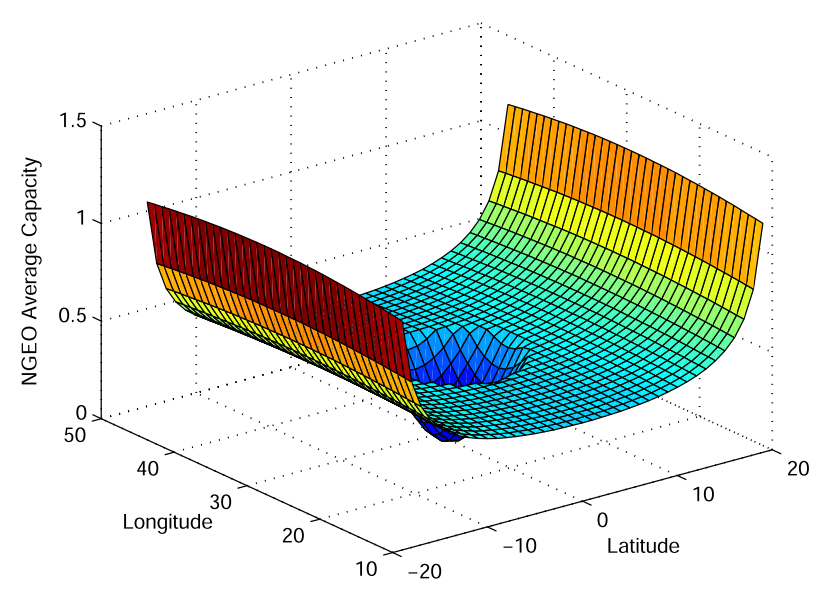

Figure 21. NGEO average capacity versus orbital position with power control at the NGEO terminal.

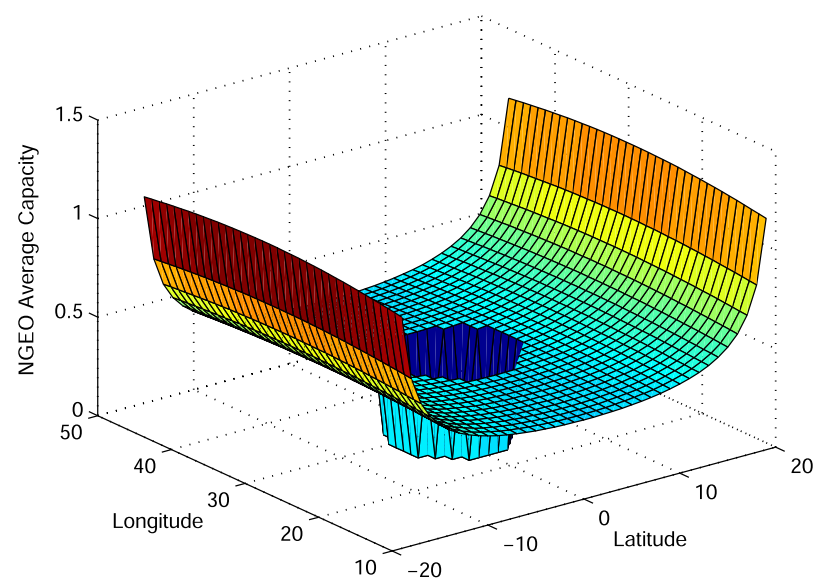

Figure 22. NGEO average capacity versus orbital position with ITU-R discrimination approach

Table V. Comparison of achievable NGEO capacity over the considered region for different cases.

\begin{tabular}{lc}
\hline Case & Value \\
\hline NGEO capacity without GEO presence & $771.29 \mathrm{bps} / \mathrm{Hz}$ \\
NGEO capacity with GEO presence and power control at NGEO terminal & $747.42 \mathrm{bps} / \mathrm{Hz}$ \\
NGEO capacity with GEO presence and ITU-R angular discrimination approach & $728.80 \mathrm{bps} / \mathrm{Hz}$ \\
\hline
\end{tabular}

the NGEO only case (without GEO presence) and the lowest for the case with discrimination anglebased approach. By implementing the proposed power control-based approach, we can achieve almost $18.62 \mathrm{bps} / \mathrm{Hz}$ more capacity than the ITU-R discrimination angle-based approach for the considered set of parameters, which is a significant gain.

\section{DISCUSSION AND FUTURE ISSUES}

Regarding the uplink coexistence of GEO and NGEO satellites in the same spectrum, the aggregate interference from NGEO terminals may be problematic to satisfy the desired interference threshold at the satellite receiver. Furthermore, in the uplink, the interference to the GEO satellite depends not only on the interference from the main lobe of the NGEO terminal, which is in-line with the GEO link, but also on the aggregate interference caused by the side-lobe gains of the beampatterns of the 
many NGEO terminals in the ground. In the downlink, there is little chance of the GEO terminals being interfered by the NGEO satellite since the main beams of the NGEO terminals and the NGEO satellite are attached in such a way that there is very low probability of overlapping the GEO terminal's main beam with the NGEO satellite's main beam. However, when the NGEO terminal is very near to the GEO terminal, especially in the equatorial region, the GEO terminal may receive significant interference from the NGEO satellite's transmission. It should be noted that if there are many NGEO satellites operating in different equatorial orbits, the GEO terminals that are located in the equatorial region may get aggregate interference from the side-lobes of the NGEO satellite's beampatterns. To facilitate the coordination between two satellite systems operating in the same spectrum, there exists regulatory constraints such as the EPFD limits of secondary transmission or the tolerable interference limits for the primary systems. In this context, the mechanisms to avoid the interference between two systems should be employed in order to protect the previously deployed GEO satellite systems while respecting the regulatory constraints.

In practice, the interference tolerance threshold for a GEO satlelite/terminal indicates the maximum permissible value of interference it can receive without any degradation in its link QoS. In other words, the aggregate interference provided by the non-GEO satellites/terminals towards a GEO terminal/satellite should be below this value. If we know the value of this parameter, we can use this in designing a suitable power control strategy for a NGEO terminal/satellite in order to protect the primary GEO satellite/terminal as illustrated in Section 4. The value of interference threshold can be fixed in such a way that the aggregated interference to noise ratio does not exceed $-6 \mathrm{~dB}[17,33]$. For example, for the user link bandwidth of $500 \mathrm{MHz}$, an interference threshold of $-123 \mathrm{dBW}$ can be considered while designing the power control strategy for a single NGEO terminal/satellite. If there exist multiple NGEO terminals/satellites, this value should be scaled accordingly in order to find out interference threshold constraint for each NGEO transmitting satellite/terminal.

The selection of interference threshold for the GEO satellite depends on the permissible level of interference based on standards or ITU-R recommendations. This interference threshold should be respected for the case of single interfering users as well as for the multiple interfering users. If there exist $N$ interfering users and they have more or less same interference towards the victim receiver, then the interference threshold can be scaled in such a way that the interference threshold that is to be protected by a single interfering user becomes $I_{T} / N$. If there is no coordination between the interfering users, the only way to respect the interference constraint of the primary receiver is by respecting the scaled amount of interference threshold by each user. The value of $N$, that is, the number of NGEO satellites operating in the same spectrum, can be known using the database of registered satellite systems. If we can allow some form of coordination between the interfering users, that is, NGEO terminals in our context, then we could allow more interference for one terminal based on traffic condition, geographical location, and so on. In the context of satellite communications, the coordination may become feasible since a single gateway is responsible for large coverage area and the gateways are generally connected with the help of a high speed link.

The main technical challenges for mitigating the interference between GEO and NGEO systems operating in the same spectrum band are provided below.

(1) Determination of the minimum separation requirement between earth stations of GEO and NGEO satellite systems based on the acceptable interference levels.

(2) Assessing the performance of GEO satellite system in the presence of in-line interference from NGEO systems.

(3) Advanced interference mitigation techniques to allow the coexistence of GEO FSS and NGEO MSS systems.

(4) Exploiting underlay and overlay cognition techniques in the coexistence of LEO/MEO and GEO satellites taking advantage of inter-satellite links between different orbits.

(5) Resource management techniques for dynamic allocation of power and carriers in two-layered satellite networks.

(6) Exploring physical layer issues in the multilayered satellite networks.

(7) To analyze and model the interference environment generated by NGEO systems properly. 
(8) Since the Ka band has also been used by terrestrial FS systems, it is an important issue to study the impact of interference on one another due to coexistence of three types of systems in the same frequency band.

\subsection{Other cognitive approaches}

5.1.1. Coordinated approach. The main concept behind this approach is that the coordination between GEO and O3b network can facilitate in spectrum sharing between two networks. The GEO gateway station and the O3b gateway station can be connected with the help of a high speed signaling link (i.e., microwave and optical fiber). In terms of the cognitive scenario, we consider multibeam GEO satellite link as the primary and the O3b satellite link as the secondary since GEO satellite is already deployed in this spectrum. We consider the GEO satellite to be multibeam satellites. With the help of the signaling link between the gateways, the O3b gateway can be aware of the beampatterns of the GEO satellite. However, the beampattern of the O3b satellite changes over the time. Since the O3b gateway has the knowledge of GEO beampattern, it can automatically select its frequency of operation not to overlap with the in-line GEO beam. If there are no more free frequencies available, the O3b can switch off its transmission on that beam when the beam passes through the the in-line center of the GEO beam. In this way, with the help of coordination between different gateways, the harmful in-line interference can be mitigated. Furthermore, based on coordination and synchronization between two systems, cognitive beamhopping system as proposed in [16] can be applied.

5.1.2. Exclusion zone plus power control approach. By finding out the proper exclusion region for the GEO earth station, the interference caused by the NGEO systems to the GEO station can be mitigated by allowing them to operate outside the EZ. Furthermore, the interference caused by the NGEO systems to the GEO satellite can be mitigated by defining the proper exclusion angle and applying the techniques such as switching, turn-off, and so on when NGEO satellites enter into the GEO exclusion angular region. However, as the number of NGEO systems increases, the above techniques do not provide better solution due to the requirement of higher spectral efficiency. In this context, different levels of EZ can be defined based on the level of interference between two systems. In the regions where interference level is too high, the only way to mitigate is either by switching transmission to another NGEO satellite or turning off the transmission. For other regions, we can apply power control to mitigate interference as described in the previous section. By combining these two approaches, the spectral efficiency can be enhanced than that of spectral efficiency obtained by using only single method.

5.1.3. Dynamic approach. In this approach, we consider O3b network and GEO links working in the normal return mode. We note that VSAT transmit-receive terminals can use the same antenna for transmission and reception purposes. We can assume similar types of terminals to be used in $\mathrm{O} 3 \mathrm{~b}$ gateways/user terminals. The concept is that the in-line interference is detected during the reception phase and the terminal does not transmit in its transmission phase until the in-line interference in the reception link does not fall below the predefined threshold. In this approach, either the O3b gateway or the terminal should be equipped with some intelligent sensor which can sense the presence of the in-line interference. As soon as it is aware of the in-line interference, it can switch off its transmission dynamically.

\section{CONCLUSION}

In this paper, a detailed overview of frequency sharing studies and interference mitigation techniques has been provided for the spectral coexistence of GEO and NGEO satellite networks. Furthermore, interference analysis has been presented for the spectral coexistence of a GEO link and an MEO link considering the $\mathrm{O} 3 \mathrm{~b}$ network as a use case, and an adaptive power control technique has been proposed in order to adapt the transmit power of the MEO satellite/terminal in order to satisfy the desired QoS of the MEO link while guaranteeing the interference to the GEO link to be below the tolerable interference limit. Moreover, several cognitive approaches such as coordinated, dynamic and combined have been discussed and future issues in this domain have been identified. We consider investigating interference 
mitigation techniques for the spectral coexistence of LEO networks considering the Iridium satellite link as a use case with other NGEO satellite networks as our future work.

\section{ACKNOWLEDGEMENTS}

This work was supported by the National Research Fund, Luxembourg under AFR (Aids Training-Research) grant for $\mathrm{PhD}$ project (Reference 3069102) and the CORE project ' $\mathrm{CO}^{2} \mathrm{SAT}$ : Cooperative and Cognitive Architectures for Satellite Networks'.

\section{REFERENCES}

1. Sharma SK, Chatzinotas S, Ottersten B. Satellite cognitive communications: interference modeling and techniques selection, 6th ASMS/SPSC Conf: Baina, Spain, Sept. 2012; 111-118.

2. Vatalaro F, Corazza G, Caini C, Ferrarelli C. Analysis of LEO, MEO, and GEO global mobile satellite systems in the presence of interference and fading, IEEE J Sel Areas Commun 1995; 132:291-300.

3. Park CS, Kang CG, Choi YS, Oh CH. Interference analysis of geostationary satellite networks in the presence of moving non-geostationary satellites, 2nd Int. Conf. Info. Technol. Convergence and Services: Cebu, Philippines, 2010; 1-5.

4. Fortes JMP, Sampaio-Neto R. An analytical method for assessing interference in interference environments involving NGSO satellite networks, Int J Satellite Commun 1999; 176:399-419.

5. Noschese P, Porfili S, Di Girolamo S. ADS-B via Iridium NEXT satellites, Tyrrhenian Int. Workshop Digital Commun. Enhanced Surveillance of Aircraft and Vehicles: Capri, Italy, 2011; 213-218.

6. Sharma SK, Chatzinotas S, Ottersten B. Exploiting polarization for spectrum sensing in cognitive SatComs, 7th Int. Conf. CROWNCOM: Stockholm, Sweden, 2012; 36-41.

7. Sharma SK, Chatzinotas S, Ottersten B. Spectrum sensing in dual polarized fading channels for cognitive SatComs, IEEE Globecom Conf:: Anaheim, CA, 2012; 3443-3448.

8. Kandeepan S, De Nardis L, Di Benedetto MG, Guidotti A, Corazza G. Cognitive satellite terrestrial radios, IEEE Globecom Conf: Miami, FL, 2010; 1-6.

9. Sharma SK, Chatzinotas S, Ottersten B. Cognitive radio techniques for satellite communication systems, IEEE Veh. Conf. Technol. (VTC-fall): Las vegas, NV, 2013; 1-5.

10. Chatzinotas S, Sharma SK, Ottersten B. Frequency packing for interference alignment-based cognitive dual satellite systems, IEEE Veh. Conf. Technol. (VTC-fall): Las vegas, NV, 2013; 1-7.

11. Suffritti R, Corazza GE, Guidotti A, Petrini V, Tarchi D, Vanelli-Coralli A, Di Renzo M. Cognitive hybrid satellite-terrestrial systems, Proc. Int. Conf. on Cognitive Radio and Advanced Spectrum Management: Barcelona, Spain, 2011; 1-6.

12. Sharma SK, Chatzinotas S, Ottersten B. Spatial filtering for underlay cognitive SatComs. In Personal Satellite Services, Social Informatics and Telecommun. Engg., Dhaou R et al (eds.), Lecture Notes of the Institute for Computer Sciences, vol. 123 Springer International Publishing, 2013, pp. 186-198.

13. Yun YH, Cho JH. An orthogonal cognitive radio for a satellite communication link, IEEE 20th Int. Symp. on Personal, Indoor and Mobile Radio Commun: Tokyo, 2009; 3154-3158.

14. Sharma SK, Chatzinotas S, Ottersten B. Interference alignment for spectral coexistence of heterogeneous networks, EURASIP J Wireless Commun and Networking 2013; 462013:1-14.

15. Sharma SK, Chatzinotas S, Ottersten B. Transmit beamforming for spectral coexistence of satellite and terrestrial networks, Int. Conf. CROWNCOM: Washington, USA, 2013; 275-281.

16. Sharma SK, Chatzinotas S, Ottersten B. Cognitive beamhopping for spectral coexistence of multibeam satellites, Future Network and Mobile Summit: Lesbon, Portugal, 2013; 1-10.

17. Sharma SK, Chatzinotas S, Ottersten B. Cognitive beamhopping for spectral coexistence of multibeam satellites, Int J Satell Commun Network March 2014. DOI: 10.1002/sat.1073.

18. Ilcev S. Low earth orbits (LEO), 20th Int. Crimean Conf. Microwave and Telecommun. Technol. (CriMiCo), 2010; 406-408.

19. Barnett RJ. O3b non-geostationary satellite system: technical information to supplement the existing schedule $S$ for the Texas gateway earth station. FCC File No. SES-LIC-20130124-00089.

20. O3b. Our technology. Online. O3b networks. (Available from: http://www.o3bnetworks.com/o3b-advantage/ our-technology) [accessed on 30 July 2013].

21. Davison EM. Spectrum issues related to satellite communications. Online. NTIA. (Available from: http://www.its.bldrdoc. gov/media/30236/dav\$_\$s.pdf.) [accessed on 25 July 2013].

22. Reed A, Posen M. Interference in the fixed satellite service bands between the feeder-links of networks using nongeostationary satellites and network using geostationary satellites, 3rd European Conf. Satellite Commun. - ECSC-3: Manchester, UK, 1993; 251-256.

23. Kobayashi H, Shinonaga H, Araki N, Ito Y. Study on interference between non-GSO MSS gateway station and GSO FSS earth station under reverse band operation, Int. Conf. Digital Satellite Commun.: Brighton, UK, 1995; 282-289.

24. Ghazvinian F, Sturza MA. Co-directional ka-band frequency sharing between non-GSO satellite networks and GSO satellite networks. (Available from: http://www.3csysco.com/Pubs/Co-Directional) [accessed on 20 July 2013].

25. TSL. Introduction to visualyse systems. Online 1996. Transfinite Systems Ltd. (Available from: http://www.transfinite.com/ content/software1.html.) [accessed on 10 August 2013].

26. Dupuis M. Use of the frequency bands $18.819 .3 \mathrm{GHz}$ and $28.629 .1 \mathrm{GHz}$ by the Fixed-Satellite Service (FSS). SAB-003-10. (Available from: http://www.ic.gc.ca/eic/site/smt-gst.nsf/eng/sf09880.html.) [accessed on 30 July 2013]. 
27. Gam H, Oh DS, Ahn DS. Effective method to assess the impact of interference between non-GSO system and fixed service, Satellite and Space Communications, IWSSC 2009. International Workshop on. September 9-11 2009;206-209.

28. Blondeau S, Maral G, Roussel T, Taisant J. Self interference in non geostationary satellite systems, Tenth Int Conf Digital Satell Commun 1995; 1:290-297, DOI 10.1049/cp:19950041.

29. Alagoz F, Korcak O, Jamalipour A. Exploring the routing strategies in next-generation satellite networks, IEEE Wireless Commun 2007; 143:79-88.

30. Di D, Qing L. A new routing algorithm of two-tier LEO/MEO mobile satellite communication systems, Communications, 2005 Asia-Pacific Conference on. Perth, WA, October 5-5 2005;111-115.

31. Nishiyama H, Kudoh D, Kato N, Kadowaki N. Load balancing and QoS provisioning based on congestion prediction for GEO/LEO hybrid satellite networks, Proc of the IEEE 2011; 9911:1998-2007, DOI 10.1109/JPROC.2011.2157885.

32. TSL. The O3b network and EPFD. Online. Transfinite Systems Ltd. (Available from: http://www.transfinite.com/papers/ O3b.pdf.) [accessed on 25 July 2013].

33. Paul A. et al. Interference proteciton criteria, phase 1-compilation from existing sources, 2005. (Available from: http:// www.ntia.doc.gov/files/ntia/publications.).

\section{AUTHORS' BIOGRAPHIES}

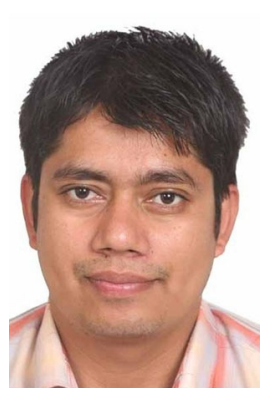

Shree Krishna Sharma received the BE degree in Electronics and Communication from Birla Institute of Technology, Mesra, India, in 2004; the MSc degree in Information and Communication Engineering from the Institute of Engineering, Pulchowk, Nepal, in 2010; the MA degree in Economics from Tribhuvan University Patan Multiple Campus, Patan, Nepal; and the MRes degree in Computing Science from Staffordshire University, Staffordshire, UK, in 2011. He is currently working toward the PhD degree with the Interdisciplinary Centre for Security, Reliability and Trust, University of Luxembourg, Luxembourg City, Luxembourg. In the past, he was also involved with Kathmandu University, Dhulikhel, Nepal, as a teaching assistant, and he served as a part-time lecturer for eight engineering colleges in Nepal. He was with Nepal Telecom for more than 4 years as a Telecom Engineer in the field of information technology an telecommunication. His research include cognitive wireless communications, resource allocation, and interference mitigation in heterogeneous wireless networks. Mr Sharma received an Indian Embassy Scholarship for his BE study, an Erasmus Mundus Scholarship for his MRes study, and an Aids Training-Research PhD grant from the National Research Fund of Luxembourg.

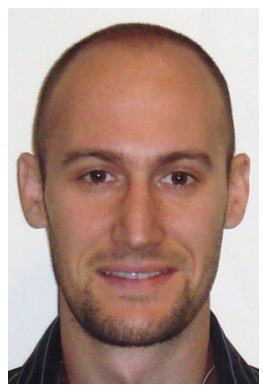

Symeon Chatzinotas received the MEng degree in Telecommunications from Aristotle University of Thessaloniki, Thessaloniki, Greece, in 2003 and the MSc and the PhD degrees in Electronic Engineering from the University of Surrey, Surrey, UK, in 2009. He is currently a research scientist in the Interdisciplinary Centre for Security, Reliability and Trust, University of Luxembourg. In the past, he has worked on numerous research and development projects for the Institute of Informatics and Telecommunications, National Center for Scientific Research Demokritos, Athens, Greece; the Institute of Telematics and Informatics, Center of Research and Technology Hellas, Thessaloniki, Greece; and the Mobile Communications Research Group, Center of Communication Systems Research, University of Surrey. He is the author of more than 110 technical papers in refereed international journals, conferences, and scientific books; and he is currently coediting a book on "Cooperative and Cognitive Satellite Systems." His research interests include multiuser information theory, cooperative and cognitive communications, and transceiver optimization for terrestrial and satellite networks.

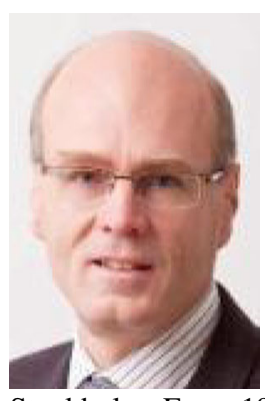

Björn Ottersten was born in Stockholm, Sweden, in 1961. He received the MS degree in Electrical Engineering and Applied Physics from Linköping University, Linköping, Sweden, in 1986 and the PhD degree in Electrical Engineering from Stanford University, Stanford, CA, in 1989. Dr Ottersten has held research positions at the Department of Electrical Engineering, Linköping University, the Information Systems Laboratory, Stanford University, the Katholieke Universiteit Leuven, Leuven, and the University of Luxembourg. In 1996/1997, he was the Director of Research at ArrayComm Inc., a start-up in San Jose, CA, based on Ottersten's patented technology. He has coauthored journal papers that received the IEEE Signal Processing Society Best Paper Award in 1993, 2001, 2006, and 2013 and three IEEE conference papers receiving Best Paper Awards. In 1991, he was appointed as the professor of signal processing at the Royal Institute of Technology (KTH), Stockholm. From 1992 to 2004, he was the head of the department for Signals, Sensors, and Systems at KTH; and from 2004 to 2008, he was the dean of the School of Electrical Engineering at KTH. Currently, he is a Director for the Interdisciplinary Centre for Security, Reliability and Trust at the University of Luxembourg. Dr 
Ottersten is a board member of the Swedish Research Council, and as Digital Champion of Luxembourg, he acts as an adviser to European Commissioner Neelie Kroes. Dr Ottersten has served as an associate editor for the IEEE Transactions on Signal Processing and on the editorial board of IEEE Signal Processing Magazine. He is currently the editor in chief of EURASIP Signal Processing Journal and a member of the editorial boards of EURASIP Journal of Applied Signal Processing and Foundations and Trends in Signal Processing. He is a fellow of the IEEE and EURASIP and a member of the IEEE Signal Processing Society Board of Governors. In 2011, he received the IEEE Signal Processing Society Technical Achievement Award. He is the first recipient of the European Research Council advanced research grant. His research interests include security and trust, reliable wireless communications, and statistical signal processing. 